\title{
Dynamics of mesoscopic systems: Non-equilibrium Green's functions approach
}

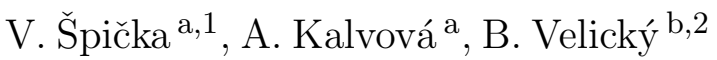 \\ ${ }^{a}$ Institute of Physics, Academy of Sciences of the Czech Republic, Na Slovance 2, 18221 Praha 8, Czech Republic \\ ${ }^{\mathrm{b}}$ Faculty of Mathematics and Physics, Charles University, Ke Karlovu 2, Praha 2, Czech Republic
}

\begin{abstract}
Recent developments in technologies and experiments enable us to observe dynamical behavior of very small quantum systems under conditions when it is sensitive to system parameters, internal interactions, the environment and the external time-dependent fields. This contribution deals with the status and perspectives of description of such truly non-equilibrium quantum many body systems using the non-equilibrium Green's functions (NGF).

The basic aim of this approach is to describe time development of the many-body system out of equilibrium from its initial state over its transient dynamics to its long time asymptotic evolution. The early stages of the transient evolution will be characterized for a broad class of the initial conditions generated in the course of Keldysh switch-on states. Gradual loss of initial correlations due to interactions and related renormalization processes in the system may, under favorable conditions, give rise to the evolution stage expressible in terms of non-equilibrium quasiparticles. This permits to reduce the description of the system behavior to a quantum transport equation. The consistency of such approximations for NGF may be judged by checking the non-equilibrium version of transport Ward identities. These identities follow from the gauge invariance of the 1st kind for NGF. To cover the whole time domain of the transient, we obtain the non-equilibrium Ward identities for NGF with initial conditions included.
\end{abstract}

Key words: Mesoscopic systems, NGF, initial conditions, correlations, Ward identities, transients PACS: 05.30.-d, 05.60.Gg, 72.10.Bg, 73.23.-b, 73.63-b

\section{Introduction}

This paper is concerned with the applications of Non-equilibrium Green's functions (NGF) [1-13] to electron transport phenomena in small (mesoscopic, nanoscopic) systems. While this field-theoretic technique has become almost standard in bulk solids, it

\footnotetext{
1 Corresponding author. E-mail: spicka@fzu.cz

2 also Center for Theoretical Study at Charles University and the ASCR, Jilská 1, 11000 Praha 1, Czech Republic
}

may said to be a minority approach in the case of small systems. This may appear as surprising, as small systems are a host of strong interactions, it is easy to bring them far from equilibrium and to induce in them fast transient processes. Transport processes in small systems are typically of a quantum nature because of strong coherence phenomena. All these features should make small systems a preferred domain of NGF. Yet this is not always the case and often methods of density matrix working in a many-particle configuration space are more convenient and powerful and lead to 
the desired results.

The underlying difference between the bulk solids and the small systems can be summed up in a few points. A bulk system is typically a (quasi-)isolated extended homogeneous solid with a finite density of electrons. It is natural to use NGF and to describe the electron transport in terms of one-particle excitations, the more so that often the non-equilibrium extension of the Landau gas of quasi-particles is a good picture of excited states of the system. Recent developments closely linking NGF with the Time Dependent Density Functional (TDDFT)[14-21] bring the NGF technique directly into the heart of modern computations. The technical difficulties with NGF are much relaxed because of the momentum representation natural for the bulk, and with the related possibility of using Wigner variables which are well suited for a quasi-classical expansion $[1,7,8]$.

A small system, by contrast, must intrinsically be open in order to participate in a transport process. The complete system - small system + extended environment(bath, leads etc.) - behaves in many ways similarly as an extended bulk, but there is a profound difference between both. All degrees of freedom can be divided into two groups: a smaller, often finite group of "relevant" degrees of freedom, and the rest taking care of decoherence, life-time effects, transport phenomena etc. The density matrix is then written for states over the configuration space of the relevant degrees of freedom. This is convenient for strongly correlated systems, for which a many-particle description is natural. For a small, very inhomogeneous, system, the momentum representation is not important, just as quasi-classical considerations.

It is now possible to indicate the space open for the use of the largely complementary approach based on NGF. First, they are congruous with tasks of finding one-particle observables, like currents and current densities, occupation numbers, spin densities. Second, they accentuate a "holistic" look on the system, permitting any strength of the coupling between the system and the environment and easily providing the true observable quantities, like the currents within the leads, for example. Third, they capture, thanks to their two-time nature, the coherence and decoherence in the system in a natural way. As a consequence, NGF appear to permit a controlled transition to simplified theories suitable for moderately fast transient processes and de- scribing the time evolution by a quantum transport equation, in this case having the form of a generalized master equation (a master equation with memory). Conditions for a further reduction to a plain master equation are also at hand. Finally, the treatment of interactions in the form of an effective one-particle theory (TDDFT) can be extended to small systems as well [19-21].

With all that in mind, we may formulate more precisely the subject of this paper: an implementation of the NGF technique in the case of a fast transient process starting at a finite initial time and induced by a non-stationary initial condition and/or by an external field turned on at the initial time. The ways of incorporating complex initial conditions in the NGF calculation are already known in several variants. This will be the first topic of this review. The initial period of a transient process is characterized by a complicated evolution known under the name of decay of initial correlations. If the external conditions are not very irregular, the system tends to a kinetic stage as the intermediate and late period of the process. The evolution during the kinetic stage is characterized by availability of simplified dynamic equations, quantum transport equations (QTE), generally speaking. This is the contents of the famous Bogolyubov conjecture $[22-24,9]$. The second topic of the paper is a general scheme, based on the so-called Reconstruction theorem [11], of reducing the complete NGF machinery to a QTE. A standard tool for this task has been one type of approximate decoupling of the particle correlation function going by name of Kadanoff-Baym Ansatz [1,810]. This Ansatz has been modified to a class of the socalled causal Ansatzes including a particularly successful Generalized Kadanoff-Baym Ansatz [25,26,8-10]. We will describe an improved version we call QuasiParticle Kadanoff-Baym Ansatz [27,10,28]. It is based on the notion of non-equilibrium quasi-particle whose meaning will be given in some detail. An important aspect of the theory are controlled approximations. We sketch here a derivation of one quantitative criterion for particle number conservation, the generalization of the transport Ward identities [29-33,7] to non-equilibrium processes of a finite duration.

To avoid misunderstanding, it should be stated that the problems discussed are posed on a general level in this paper.

First, all results are equally valid for the bulk and 
for the small systems. In a parallel communication in this volume [34], we treat a specific nanoscopic system, a molecular bridge between two leads, using the same general approach, but going to formal elaboration of the NGF in detail.

Second, we do not use any specific physical approximation to treat the interactions, couplings etc. There are several general properties of the self-energy which we assume and which would have to be verified in each specific instance. These include the self-consistent nature of the approximations and the existence of two characteristic times, the quasi-particle formation time and the collision duration time, which should define the shortest time scale entering the physical problem. On the formal side, we keep the universality of the equations by avoiding any specific representation, so that the Green's functions and related quantities appear mostly as having only time arguments explicit. Otherwise, they should be viewed as operators acting on one-particle states.

Before giving an outline of the paper in some detail, one particular topic - central to the whole paper deserves a more extended discussion: the incorporation of initial correlations into the NGF formalism.

The initial correlations enter the NGF with finite initial time

$$
\left.G(1,2)=-\mathrm{i}\left\langle\boldsymbol{T}_{\mathrm{c}}\left\{\psi(1) \psi^{\dagger}(2)\right)\right\}\right\rangle_{\mathrm{I}},
$$

through the statistical average over the many-body initial state indicated by the ${ }_{\text {I }}$ label.(Full definition ... Eq. (1), time trajectories Fig. 1.) This straightforward definition is fully general and encompassing all physically admissible situations. It has two clear drawbacks, which make it barely practicable. First, the information contained in an arbitrary many-particle statistical operator is excessive, as most of the details of the correlations get lost in the process of averaging and only a heavily reduced amount of information is relevant. Second, for an arbitrary initial state, the average is difficult to perform, as the Wick theorem does not hold, unless the initial state is uncorrelated. The problem of initial correlations has a long history in the literature $[35-45,19]$ but we only intend to sketch the presently prevailing ways of attacking it. Interestingly enough, there are two main directions in the field, each having as its basic point of departure one of the two difficulties.
In the first approach [40-45], correlations in the initial state are represented by a chain of equal-time correlation functions for particle complexes. The equations of motion for the NGF can be written as an expansion in terms of these correlation functions and, with a suitable termination, the NGF becomes available. It was possible to establish a consistency criterion between the physical approximation for the NGF for all times and the required correlation functions at the initial time. In particular, for the T-matrix approximation for the particle-particle interaction, the proper initial state information was contained in the pair correlation function. We have proposed to denote this type of approach as a synchronous technique of treating the initial correlations.

In the second approach $[38,39,19]$ the basic idea has been to augment the basic finite initial time Keldysh trajectory by an additional time interval, over which the correlated initial state would evolve from an auxiliary uncorrelated state. In the basic version, proposed by Danielewicz [38], the initial state was a fully correlated equilibrium state which was obtained by a Matsubara-like expansion on an imaginary time interval. The time arguments of the Green's function had to be taken both rom the real and the imaginary domain and this complete Green's function incorporated the correlations of the true initial state. For its evaluation, the auxiliary uncorrelated state was used to warrant applicability of the Wick theorem. This concept was further extended by Wagner [39] to arbitrary initial states. This technique has been widely used since. To contrast it with the first approach, we proposed to call this technique diachronous.

There is an interesting complementarity between both approaches. In the synchronous method, equaltime correlations of ever increasing complexity are employed. By contrast, in the diachronous methods, the object of study is just the one particle Green's function. However, in order to capture the initial correlations properly, this Green's function has to be known over a whole interval of times surrounding the initial time.

We employ in this review the diachronous approach in a modified version: instead of extending the original finite-time loop in a Matsubara-like manner [7], it is possible to augment the loop by pushing its lower limits to $-\infty$. If the initial state at this remote initial time is taken as uncorrelated, we have to deal with the orig- 
inal Keldysh initial conditions [2]. Turning the correlations on in an adiabatic way, we may achieve the correlated initial state just at the initial time $t_{\mathrm{I}}$ and continue with a process of physical interest. That may appear as side-stepping from the problem of initial correlations altogether. In fact, however, the NGF solution for the extended loop can be employed to derive from it the finite-time self-energy including the initial correlations for our process in a closed form. This means that both diachronous techniques lead to the same result. It will be convenient to speak of a host process, which is the complete process starting from the Keldysh initial condition at $-\infty$ and of an embedded physical process, or embedded transient starting at $t_{\mathrm{I}}$. If the decay of initial correlations is fast, the working scheme based on the link between the host and the embedded process becomes useful, as will be explained at some length below.

The paper is organized as follows: After this Introduction, the NGF with a finite initial time are defined on the Schwinger-Keldysh contour, rewritten in the matrix form and the Dyson equations are given in our notation for the self-energies including the singular parts of $\Sigma^{<}\{$Sec. 2$\}$. Then the problem of quantum transport equations is addressed from a general point of view. First, the precursor quantum transport equation including the initial correlations is given and the Bogolyubov conjecture about a fast decay of initial correlations is presented. The precursor equation can be converted into a true transport equation with the aid of the so-called Generalized Kadanoff-Baym Ansatz. An exact counterpart of the Ansatz are reconstruction equations. We present this subject matter first for the original case of uncorrelated initial conditions, which is reasonably well understood. A possibility of solving the Dyson equation for the particle correlation function in a cycle between the precursor equation and the reconstruction equations is summarized into the reconstruction theorem. We are interested in the inclusion of the correlated initial conditions; this case is covered by a newly formulated Generalized reconstruction theorem: The generalized reconstruction cycle is not truly closed in itself, as it requires an input concerning the correlated initial conditions $\{\mathrm{Sec} .3\}$. The possible practical ways of incorporation of the initial many body state with correlations have been characterised above. We concentrate on the method of embedding the finite process in a host process whose preparation stage serves to create an initial state, in general out of equilibrium and including the initial correlations. As the host process we propose to use the tractable Keldysh switch-on states. For systems with their self-energy concentrated to a narrow strip around the equal-time diagonal in the plane of two time variables, the width of the strip can be introduced as a characteristic time $\tau^{\star}$ and identified with the Bogolyubov time measuring the decay of correlations $\{$ Sec. 4$\}$. Then, the problem of QTE with initial conditions can be addressed once more. The Bogolyubov conjecture is confirmed and it is shown that the initial correlations decay first. The system is then governed by a QTE. After a period comparable with the relaxation time, the initial conditions are forgotten and a true kinetic stage with a QTE and gliding initial conditions ensues $\{\mathrm{Sec} .5\}$. In practice, a reduction of the full reconstruction cycle to a QTE has mostly been connected with the use of GKBA. This is summarized as a starting point to improved approximations based on the use of non-equilibrium quasi-particles. For external fields changing at a moderate rate, these entities exist and permit to rearrange the reconstruction equations to a renormalized form showing that a Quasi-particle Kadanoff-Baym Ansatz may be a better choice for developing the QTE. This leads to a generalization to a class of causal Ansatzes and to the question of their comparison, qualitative and quantitative $\{$ Sec.6 $\}$. One of the quantitative criteria for judging various approximations in the transport theory is the validity of transport Ward identities which in turn are linked with the particle number conservation. In the last section, we derive the Ward identity for NGF in the case of an arbitrary non-equilibrium process starting from a correlated initial state. It is shown that the exact reconstruction equations are a necessary condition for the Ward identity to hold $\{$ Sec. 7$\}$.

\section{NGF with initial conditions}

\subsection{NGF- basic definitions}

The dynamics of the system is defined by the full Hamiltonian $\mathcal{H}+\mathcal{U}(t)$ consisting of the system Hamiltonian $\mathcal{H}$ and an additive external disturbance $\mathcal{U}(t)$. A dynamical process is described by the statistical operator $\mathcal{P}(t)$ which is fully specified by its initial 
state $\mathcal{P}_{\mathrm{I}}$ at $t=t_{\mathrm{I}}$ (an arbitrary equilibrium or out-ofequilibrium state).

The Non-equilibrium Green's Function is defined in the usual manner [8-11],

$$
G(1,2)=-\mathrm{i} \operatorname{Tr}\left(\mathcal{P}_{\mathrm{I}} \boldsymbol{T}_{\mathrm{c}}\left\{\psi\left(1 \mid t_{\mathrm{I}}\right) \psi^{\dagger}\left(2 \mid t_{\mathrm{I}}\right)\right\}\right)
$$

with the Heisenberg field operators $\psi, \psi^{\dagger}$ anchored at $t_{\mathrm{I}}$ and the time-ordering operator $\boldsymbol{T}_{\mathrm{c}}$ acting along the closed time path $\mathbb{C}$ extending from $t_{\mathrm{I}}$ to $+\infty$ and back. To indicate the initial time, we occasionally write $G_{t_{\mathrm{I}}}(1,2)$ or $G_{\mathrm{I}}(1,2)$ instead of $G(1,2)$ and $\mathbb{C}_{\mathrm{I}}$ instead of $\mathbb{C}$. We will use this convention when working with two initial times at once.

We represent the contour-ordered NGF, following Keldysh $[2,5]$, by a $2 \times 2$ matrix GF of real time, employing the Langreth-Wilkins matrix $[3,4,8,10,11]$ having three components, the less-correlation function $G^{<}$and two (equivalent) propagators, $G^{A}(1,2)=$ $\left[G^{R}(2,1)\right]^{\dagger}$ :

$$
\mathbf{G}=\left\|\begin{array}{cc}
G^{R} & G^{<} \\
0 & G^{A}
\end{array}\right\|
$$

and similarly for the self-energy and other quantities.

\subsection{Dyson equation with initial conditions}

It is convenient to write the Dyson equation first by components. For the propagators, it will have the form

$$
G^{R, A}=G_{0}^{R, A}+G_{0}^{R, A} \Sigma^{R, A} G^{R, A}, \text { etc. }
$$

in which the self-energies are "regular" two-time functions. The mean-field "singular" parts of the selfenergy are included into the free propagators. The less-component of the Dyson equation has the form $[38,11]$

$$
\begin{aligned}
& G^{<}=G^{R} \Xi^{<} G^{A}, \\
& \Xi^{<}={ }_{\circ} \Sigma_{\circ}^{<}+{ }_{\circ} \Sigma_{\bullet}^{<}+._{\Sigma_{\circ}^{<}}^{<} ._{\Sigma_{\bullet}}^{<}
\end{aligned}
$$

The integrations in (4) start at $t_{\mathrm{I}}$. The four terms have a varying degree of singularity at the initial time. The open circles indicate a time variable fixed at $t_{\mathrm{I}}$, the filled ones a time variable continuous in $\left(t_{\mathrm{I}}, \infty\right)$. The regular term.$_{.}^{<}$corresponds to the Dyson equation as it is usually written for $t_{\mathrm{I}} \rightarrow-\infty$, namely $G^{<}=$ $G^{R} \Sigma^{<} G^{A}$. The other terms play each a specific role. In particular, ${ }_{\circ} \Sigma_{\bullet}^{<}$and.$_{\Sigma_{\circ}}^{<}\left(\Sigma^{c}, \Sigma_{\mathrm{c}}\right.$ of [38]) are related to the initial correlations. They have the form

$$
\begin{aligned}
& . \Sigma_{\circ}^{<}\left(t, t^{\prime}\right)=\Lambda_{\mathrm{O}}^{<}\left(t, t_{\mathrm{I}}\right) \delta\left(t^{\prime}-t_{\mathrm{I}}^{-}\right), \\
& { }_{{ }_{\mathrm{o}} \Sigma_{\bullet}^{<}\left(t, t^{\prime}\right)={ }_{\mathrm{o}} \Lambda^{<}\left(t_{\mathrm{I}}, t^{\prime}\right) \delta\left(t-t_{\mathrm{I}}^{-}\right)} t_{\mathrm{I}}^{-}=t_{\mathrm{I}}-0
\end{aligned}
$$

and are thus equivalent to single-time continuous functions $\Lambda_{\mathrm{o}}^{<}\left(t, t_{\mathrm{I}}\right)$, ${ }_{\mathrm{o}} \Lambda^{<}\left(t_{\mathrm{I}}, t^{\prime}\right)$ dependent on $t_{\mathrm{I}}$ as on a parameter. For the correlated initial conditions, these two functions must be determined in addition to the regular less self-energy.

The last term,

$$
\begin{aligned}
& { }_{\circ} \Sigma_{\circ}^{<}\left(t, t^{\prime}\right)=\mathrm{i} \rho\left(t_{\mathrm{I}}\right) \delta\left(t-t_{\mathrm{I}}^{-}\right) \delta\left(t^{\prime}-t_{\mathrm{I}}^{-}\right) \\
& \rho(t)=-\mathrm{i} G^{<}(t, t)
\end{aligned}
$$

represents the uncorrelated part of the initial conditions. This is the only part of $\Xi^{<}$which enters the free particle correlation function

$$
G_{0}^{<}=G_{0}^{R}{ }_{\circ} \Sigma_{\circ}^{<} G_{0}^{A}
$$

The Dyson equation for the whole GF matrix (2) reads simply

$$
\mathbf{G}=\mathbf{G}_{0}+\mathbf{G}_{0} \boldsymbol{\Sigma} \mathbf{G}
$$

if we define the free GF and the self-energy by

$$
\mathbf{G}_{0}=\left\|\begin{array}{cc}
G_{0}^{R} & G_{0}^{<} \\
0 & G_{0}^{A}
\end{array}\right\|, \quad \boldsymbol{\Sigma}=\left\|\begin{array}{cc}
\Sigma^{R} & \Sigma^{<} \\
0 & \Sigma^{A}
\end{array}\right\|
$$

where the self-energy incorporates the singular initialcorrelation components:

$$
\Sigma^{<} \equiv \Xi^{<}-{ }_{0} \Sigma_{0}^{<}=._{0} \Sigma_{0}^{<}+{ }_{0} \Sigma_{\bullet}^{<}+._{\bullet}^{<}
$$

Written by components, Eq. (8) reproduces (3) and (4).

\section{Quantum transport out of equilibrium with finite time initial conditions}

Any quantum transport problem can be considered as solved, of course, if the Non-equilibrium Green's Function for the underlying physical process is determined directly from the equations of motion. This approach is slowly becoming routine, at least for several important classes of problems; in some cases, readymade solvers for a numerical elaboration of the problem are available. This does not diminish the importance 
of reducing the full NGF machinery to quantum transport equations. Earlier the only viable route to finding explicit solutions, use of the quantum transport equations offers even today an easier and more physically transparent approach to complex problems of quantum transport. Much insight is gained in the process of deriving the transport equations from the Green's functions point of view, which permits to establish well defined criteria for such transformation.

The background and a point of departure for such work is provided by the Bogolyubov conjecture [22$24,9]$ which claims that a process starting from an arbitrarily correlated non-equilibrium state at a finite initial time enters the kinetic stage of evolution immediately after the decay of initial correlations, that is beyond an initial period lasting a characteristic decay time $\tau_{\mathrm{B}}^{\star}$. The kinetic regime is defined as such, in which the dynamics of the system is governed by a closed quantum transport equation for the one-particle density matrix. This conjecture is a corrolary of the general principle of the decay of correlations; even so, it remains, in its original form, rather an intuitive guess than a firmly established statement.

The purpose of this section is to modify and extend the standard route to the quantum transport equations (QTE) using the formal means of the NGF so as to incorporate the finite time initial conditions. In the following section, we use the developed formal means to query the Bogolyubov conjecture. In particular, we demonstrate that for any initial state generated by a preparation process, the Bogolyubov conjecture can be proved assuming that a finite $\tau_{\mathrm{B}}^{\star}$ time exists and is the shortest characteristic time for a given system undergoing a given non-equilibrium process.

\subsection{Precursor quantum transport equation and the Bogolyubov Conjecture}

The well known starting point on the way from NGF to transport equations is the precursor transport equation (called also the Generalized Kadanoff-Baym Equation). It is a differential equation obtained from Eq. (4), and Eq. (3) written as $\left[G^{R, A}\right]^{-1}=\left[G_{0}^{R, A}\right]^{-1}-\Sigma^{R, A}$. It already has a structure closely related to transport equations:

$$
\begin{aligned}
G_{0}^{-1} G^{<}-G^{<} G_{0}^{-1}= & \Sigma^{R} G^{<}-G^{<} \Sigma^{A} \\
& -G^{R} \Sigma^{<}+\Sigma^{<} G^{A}
\end{aligned}
$$

To obtain a single time transport equation the external times are set equal, $t=t^{\prime}$. The l.h.s. of the last equation then represents an unrenormalized drift of the one-particle density matrix $\rho$ :

$$
\text { 1.h.s. of }(11) \stackrel{t=t^{\prime}}{\longrightarrow} \frac{\partial \rho}{\partial t}+\mathrm{i}\left[H_{0}, \rho\right]_{-}
$$

This has the form proper for a QTE in which $H_{0}(t)$ is the mean field one-particle Hamiltonian.

By contrast, the r.h.s. of (11) consists of four "generalized collision" terms involving double time less quantities even for $t=t^{\prime}$. The one-particle density matrix does not enter these expressions. For a finite initial time, $\Sigma^{<}$incorporates, in addition, the initial correlations according to Eq. (10), which are not expressed in terms of $\rho$ either.

The Bogolyubov conjecture as applied specifically to this NGF equation demands thus two things: $\diamond$ First, a characteristic time $\tau_{\mathrm{B}}^{\star}$ has to exist such that all terms and corrections explicitly respecting initial correlations vanish for $t, t^{\prime}>t_{0}+\tau_{\mathrm{B}}^{\star}$. The solutions of the precursor equation then depend on the initial correlations but implicitly, through the initial evolution preceding $t_{0}+\tau_{\mathrm{B}}^{\star} . \diamond$ Second, the precursor transport equation has to permit a conversion to the (quantum) transport equation proper by expressing all less quantities in terms of the one particle density matrix and propagators. The latter take over the responsibility for all coherence and memory effects and constitute together the generalized collision terms. The one particle density matrix remains as the (only) unknown on both sides of Eq. (11). This transformation to a transport equation is fully in the spirit of the Bogolyubov conjecture, which thus gets a more definite formal expression, suitable for further treatment.

Well known approximations devised in this context under the name of "Ansatzes", starting from the Kadanoff-Baym Ansatz [1] have been introduced with success long time ago [8,9]. More recently, an exact reformulation of the Ansatz strategy under the name of Reconstruction theorems has been undertaken [811]. These techniques and their extension taking into account the initial correlations will be discussed in the next two sections. 
3.2. Reconstruction equations and the problem of QTE without initial correlations

In this sub-section, we summarize the method of generating a QTE from the precursor transport equation for the standard case of the Keldysh initial conditions at $t_{\mathrm{I}} \rightarrow-\infty$. It will be apparent that the same derivation would hold for NGF with finite initial time $t_{\mathrm{I}}$, but uncorrelated initial conditions.

Two separate approximations involved in this procedure have a rather different nature:

1. The double-time $G^{<}\left(t, t^{\prime}\right)$ is replaced by an expression involving only its time diagonal $\rho(t)=-\mathrm{i} G^{<}(t, t)$ and propagators. Two approximate replacements have become known under the label of "Ansatzes". The historically first one has been the famous Kadanoff-Baym Ansatz $[1,8]$, schematically $G^{<}=\frac{1}{2}\left[\rho, G^{A}-G^{R}\right]_{+}$. This Ansatz is suited for transport in fields slowly varying both in space and time (... quasiclassical and adiabatic limit). We will concentrate on its modification, the Generalized Kadanoff-Baym Ansatz [25,26,8-11]

$$
G^{<}=-G^{R} \rho+\rho G^{A}
$$

which has been proposed for fields which vary smoothly in time, but on a scale comparable to the intrinsic relaxation times of the system, so that the evolution is non-adiabatic. This will typically be the case of fast transients.

2. For the self-energy, a self-consistent approximation specifying it in terms of the Green's function is assumed:

$$
\boldsymbol{\Sigma}=\boldsymbol{\Sigma}[\mathbf{G}]
$$

This assumption is justified for the uncorrelated initial condition, whether at an infinitely remote initial time, or at a finite one, at least to all orders of the perturbation theory.

The approximation 1. for $G^{<}$is also introduced into this expression. All of that enters Eq. (11). A quantum transport equation results whose details depend both on the "physical" approximation (14) and on the approximate replacement for $G^{<}$, elected to be Eq. (13) in the present case. It should be noted that the quasiclassical approximation commonly invoked in derivations of Boltzmann-like transport equations does not enter the present scheme up to now. It would be another step depending on another small parameter.

The approximate steps $\mathbf{1}$. and $\mathbf{2}$. are controlled to a large degree independently. This has led to the so- called Reconstruction theorem according to which the full Dyson equation for $G^{<}$, for the self energy given by a specific prescription (14), can be transformed to an equivalent system of coupled equations: the GKBE (11) on the one hand, and the so-called reconstruction equations (RE) $[25,8,10,11]$ replacing the Ansatz (13) on the other hand. This gives rise to a systematic QTE scheme, in which the GKBA is but a zeroth order approximation and can be improved by iteration. We show an explicit form of these equations right for the correlated initial condition in the following section.

\subsection{Generalized reconstruction theorem: correlated initial state}

In the case of a correlated initial state at a finite initial time, the process is similar, but more involved. We start right away from the exact reconstruction equations for $G^{<}$extended so as to incorporate the finite time initial conditions.

1bis. The reconstruction equations are an exact consequence of the Dyson equation (4). They have the appearance of an integral equation serving to reconstruct full $G^{<}$from the "free term" which coincides with the Ansatz expression (13),

$$
\begin{array}{rlr}
G^{<}\left(t, t^{\prime}\right)= & -G^{R}\left(t, t^{\prime}\right) \rho\left(t^{\prime}\right) & t \geq t^{\prime} \geq t_{\mathrm{I}} \\
& +\int_{t^{\prime}}^{t} \mathrm{~d} \bar{t} \int_{t_{\mathrm{I}}}^{t^{\prime}} \mathrm{d} \overline{\bar{t}} G^{R}(t, \bar{t}) \Sigma^{R}(\bar{t}, \overline{\bar{t}}) G^{<}\left(\overline{\bar{t}}, t^{\prime}\right) \\
& +\int_{t^{\prime}}^{t} \mathrm{~d} \bar{t} \int_{t_{\mathrm{I}}}^{t^{\prime}} \mathrm{d} \overline{\bar{t}} G^{R}(t, \bar{t}) \Xi^{<}(\bar{t}, \overline{\bar{t}}) G^{A}\left(\overline{\bar{t}}, t^{\prime}\right) \\
G^{<}\left(t, t^{\prime}\right)= & \rho\left(t^{\prime}\right) G^{A}\left(t, t^{\prime}\right)+\cdots & t^{\prime} \geq t \geq t_{\mathrm{I}}
\end{array}
$$

In other words, Eq. (15) serves to reconstruct a full double time function from the knowledge of its time diagonal i $\rho$ (and propagators). Unfortunately, the integral terms involve also the $\Xi^{<}$self-energy, see (4), with all initial time corrections and this is a problem in general. In the uncorrelated case, these corrections vanish and the self energy can be obtained from Eq. (14). This defines the reconstruction procedure of the preceding section. 
Returning to the correlated case, if the integrals in (15) were neglected, this equation would simplify just to the Generalized Kadanoff-Baym Ansatz, and the reconstruction would be immediate, but approximate. The Ansatz thus neglects, among other things, all dependence on the initial conditions and may be too coarse an approximation in this sense. This points rather to subtlety of the initial transient than to a drawback of the Ansatz, which has been conceived from the outset for use in a fully developed quantum kinetic regime.

2bis. The difficulty mentioned in point $\mathbf{1 b i s}$. concerns both the reconstruction equation (15) and the precursor equation (11): with a finite initial time, there is no analogue of Eq. (14) available. This is related to the absence of the Wick theorem etc., as stressed in the Introduction. Even if the Ansatz were used for $G^{<}$and so the difficulty were circumvented in (15), it would remain in the r.h.s. of the precursor equation: we have no autonomous access to the self-energy terms. This means that, in general, the reconstruction theorem cannot be readily transferred to the case of initial correlations and calls for a substantial generalization.

The generalized Reconstruction theorem states that the solution of the Dyson equation for $G^{<}$can be obtained according to the scheme

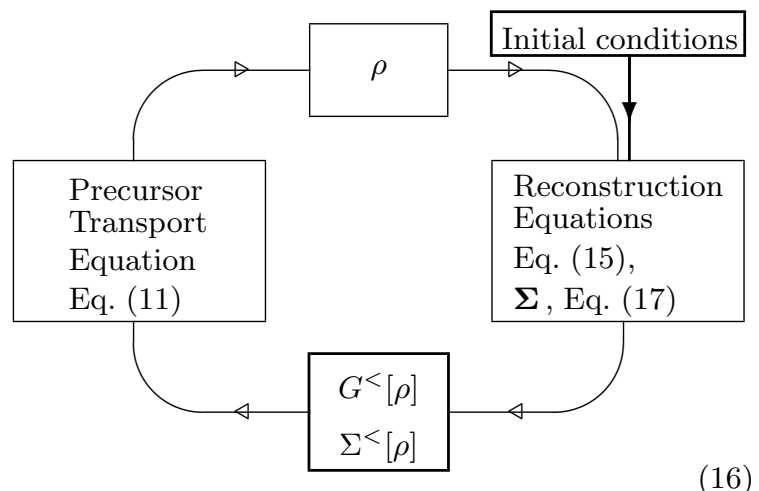

The core of the scheme is the cycle indicated by open arrows. The cycle follows the true physical process under investigation. It starts at $t_{\mathrm{I}}$ and is incremental in time. The initial conditions - correlated or uncorrelated alike - have to be fed in from outside, as shown symbolically by a box "Initial conditions" in the top right corner of Eq. (16).

We have to distinguish the uncorrelated initial con- dition from the correlated one, however. The uncorrelated initial conditions shrink just to the initial oneparticle density matrix which is known. This would be enough to reconstruct the initial correlation function and its self-energy in terms of initial $\rho$ (right hand box). These would enter the precursor transport equation and the result would be $\rho$ for the first incremental time (left hand box). This cycle would then proceed on and on. This is the practical use of the reconstruction theorem, by which the Dyson equation is solved for all times using the already known section of $\rho$ as a function of time as an intermediary.

In the correlated case, the initial conditions which have to be input during the cycle in addition to $\rho_{\mathrm{I}}$ involve basically the initial time corrections to $\Xi^{<}$. The reconstruction cycle may become practicable, if it is supplemented by the corresponding algorithm. As outlined in the Introduction and will be described in detail in the next section, one possibility is to view the transient starting at $t_{\mathrm{I}}$ as embedded in a host process starting from the Keldysh initial conditions. We describe the details of the approach in the next section. Anticipating the result, we may assume that a rule exists yielding the self-energy (9), (10) as a functional of the augmented Green's function $\mathbf{G}_{-\infty}$,

$$
\boldsymbol{\Sigma}=\boldsymbol{\Sigma}\left[\mathbf{G}_{-\infty}\right]
$$

in the notation of Fig. 1. This relation appears as exceedingly symbolic, but this is just right in the scheme (16).

The replacement of the Dyson equation by the reconstruction cycle might become useful, if there was a way of expressing the r.h.s. of the precursor equation in a compact form of "generalized collision terms" and so the cycle would shrink to solving the resulting quantum transport equation directly - in the kinetic stage of the evolution. The box decisive for this in the whole cycle is thus the prescription $\rho \rightarrow G^{<}, \Sigma^{<}$marked by a heavy frame at the bottom of the diagram. Even the simplified collision terms in the transport equation would have to be calculated over and over again for a transient, because they have a memory and depend on time. We will continue the discussion of transport equations in Sec. 5, returning to the correlated initial conditions in Sec. 5.1 and to the kinetic stage in Secs. 5.2 and 6 . Before that, however, we have to bring in two general prerequisites: incorporation of the initial conditions in the NGF formalism and extraction of prop- 
erties of the self-energy salient for establishing and using the transport equations.

\section{Initial conditions for a transient embedded in a host process}

The correlated initial conditions call for a full NGF treatment of a transient in general; it has to be augmented by some of the techniques outlined in the Introduction. The effect of the initial correlations will gradually diminish and the kinetic stage may ensue, but any use of quantum kinetic equations during the early stages of a transient should be considered with an extreme caution.

Several techniques of incorporating the initial conditions for NGF have been listed in the Introduction. Presently, we restrict ourselves to the diachronous techniques, in which the NGF is determined in a time domain extending the time span of the transient process; the two choices of extending the time axis to times preceding the initial time $t_{\mathrm{I}}$ are shown in Fig. 1. One would be the formal extension of the $\mathbb{C}_{\mathrm{I}}$ loop by an imaginary time interval $\left[t_{\mathrm{I}}, t_{\mathrm{I}}-\mathrm{i} \tau\right]$ to the $\mathbb{C}_{\mathrm{M}}$ trajectory as introduced in $[38,39]$. We will pursue the other possibility of extending the finite loop, namely to an infinite Keldysh loop $\mathbb{C}_{-\infty}$ starting and ending at an infinitely remote past with a U-turn at $+\infty$. This will be described in the next section. For an actual use of the Danielewicz-Wagner technique see [19].

\subsection{Keldysh switch-on processes}

First a terminological note: under the Keldysh initial condition, we will understand an uncorrelated initial state at $-\infty$. It need not be an equilibrium state. For example, it may describe uncoupled leads with a mutual bias, or even with a temperature difference [46]. There is also the Keldysh initial condition at a finite initial time. This is uncorrelated again. This case is characterized by reducing the full initial condition to the initial one-particle density matrix $\rho_{\text {I }}$, see Eq. (6). The expressions for NGF with initial conditions found in the literature are often equivalent with this uncorrelated case: $[7,8]$.

Returning to the infinite Schwinger-Keldysh contour, it can accommodate an extremely rich class of

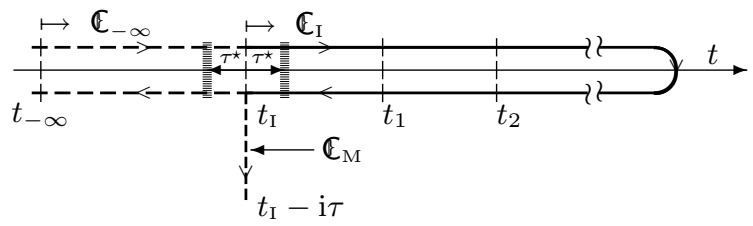

Fig. 1. A finite-time Schwinger-Keldysh contour and its diachronous extensions. $\mathbb{E}_{\mathrm{I}}$ : The closed time ordering contour for a transient starts at the initial time $t_{\mathrm{I}}$, goes to $+\infty$ and returns to $t_{\mathrm{I}}$. $\mathbb{C}_{\mathrm{M}}$ : Extension by a Matsubara-like imaginary time interval according to $[7,38]$ or $[39,19]$. $\mathbb{C}_{-\infty}:$ Extension of $\mathbb{C}_{\mathrm{I}}$ along the time axis to the past. The resulting contour starts at $t_{-\infty}$. The dashed segment preceding $t_{\mathrm{I}}$ accommodates the preparation process. The two adjoining processes form together the host process, in which the transient is embedded. For $t_{-\infty} \rightarrow-\infty$, the host process with an uncorrelated initial condition becomes a Keldysh switch-on process. Two diffuse boundaries bracketing $t_{\mathrm{I}}$ at the distance $\sim \tau^{\star}$ : If a finite correlation decay time $\tau^{\star}$ exists, the lower boundary indicates the depth of coupling between the preparation process and the transient, the upper one marks the extent of penetration of the initial correlations into the future, see Secs. 4.4 and 5.

processes bearing the generic name of the Keldysh switch-on processes. These processes start from general uncorrelated states, become correlated as the interactions are switched on and are driven by an endless variety of external influences. It may be said that the intermediate states passed through by the system in the course of such processes form in their entirety the class of all physically attainable states of the system, including states out of equilibrium and incorporating correlations of widely different nature and strength. Any of these states may be used as an initial state for the transient process we wish to study. Thus, we can start the transient from "all" physically meaningful initial states. Still, the Wagner method admits, in principle at least, quite arbitrary initial states, including those which are artificially overcorrelated etc. Such states fall out of our scope by definition.

It remains to describe the technical means of using these switch-on states as correlated initial states; the technique we call, in physical terms, embedding of the transient in a host switch-on process. The formal tool is a variant of the partitioning (folding down) method [47], the partitioning in time [48]. 
4.2. A transient embedded in a Keldysh switch-on process

In the treatment proposed here, the initial conditions for the transient are not arbitrary, but must result from the previous history of the system. The transient process is in this sense embedded in a much longer "host" process as its late part commencing at $t_{\mathrm{I}}$. The correlated initial conditions right at $t_{\mathrm{I}}$ are then constructed as an outcome of preparation, that is the antecedent period of the host process: Right from the start of the cycle (16) concerning times $t \geq t_{\mathrm{I}}$ we have to take into account the evolution preceding the initial time. As stressed already in the Introduction, this is the ultimate of a diachronous approach to initial correlations: it is enough to know no more than the one-particle GF, but over all the infinite past in the general case. The cycle of Eq. (16) is not autonomous in this sense, because we have to feed in the information about the preparation stage in order to generate the self-energy incorporating the initial correlations. That does not look as more useful than working with the complete host process directly: the relevant Green's function has to be known beforehand anyway. We will show, however, how an advantageous finite-time scheme of accounting for the initial correlations can be set up for systems with quickly decaying correlations.

Now, we turn this basic formulation into the formal task of determining explicit expressions for the NGF with an initial time $t_{\mathrm{I}}$, at which the correlated initial state is $\mathcal{P}_{\mathrm{I}}$. This initial state will not be arbitrary, as said above, but it will coincide with the state at which an antecedent ("preparation") process will have arrived at the initial time. Beyond that time, the evolution will continue as the dynamical process in question ("measurement" or "observation").

This interpretation of the two stages as the past and the future with respect to the initial time is entirely subjective and our task is, in fact, to compare two processes differing only in their time definition range, see Fig. 1. The process under study evolves along the Schwinger-Keldysh trajectory $\mathbb{C}_{\mathrm{I}}$ starting and ending at $t_{\mathrm{I}}$ and is described by the Green's function we denote $\mathbf{G}$ suppressing the subscript I . This process is augmented by a preparation stage running between $t_{-\infty}$ and $t_{\mathrm{I}}$. Together, an extended host process results with the $\mathbb{C}_{-\infty}$ trajectory, and $\mathbf{G}_{-\infty}$ the associated NGF .
The $\mathbb{C}_{\mathrm{I}}$ process is embedded in the $\mathbb{C}_{\infty}$ one. Both processes describe the same evolution beyond $t_{\mathrm{I}}$. This coincidence permits to build up the NGF of the short process starting from that of the long process. Once this is done, the $t_{\mathrm{I}}$ process may be viewed as an autonomous "restart" process which is being restarted from a frozen initial state $\mathcal{P}_{\mathrm{I}}=\mathcal{P}_{-\infty}\left(t_{\mathrm{I}}\right)$ at $t_{\mathrm{I}}$.

\subsection{Self-energy for an embedded transient}

The core of this section will be the construction of explicit expressions for the $\Xi^{<}$self-energy entering the Dyson equation (4) for the "embedded" block of $G^{<}$ as derived from the complete switch-on process by the time-partitioning method [48]. First, Eq. (4) will be written in a more explicit form:

$$
\begin{gathered}
G^{<}\left(t, t^{\prime}\right)=\mathrm{i} G^{R}\left(t, t_{\mathrm{I}}\right) \rho\left(t_{\mathrm{I}}\right) G^{A}\left(t_{\mathrm{I}}, t^{\prime}\right) \\
+G^{R}\left(t, t_{\mathrm{I}}\right) \times \int_{t_{\mathrm{I}}}^{t^{\prime}} \mathrm{d} u{ }_{\mathrm{o}} \Lambda^{<}\left(t_{\mathrm{I}}, u\right) G^{A}\left(u, t^{\prime}\right) \\
+\int_{t_{\mathrm{I}}}^{t} \mathrm{~d} v G^{R}(t, v) \Lambda_{\mathrm{o}}^{<}\left(v, t_{\mathrm{I}}\right) \times G^{A}\left(t_{\mathrm{I}}, t^{\prime}\right) \\
+\int_{t_{\mathrm{I}}}^{t} \mathrm{~d} v \int_{t_{\mathrm{I}}}^{t^{\prime}} \mathrm{d} u G^{R}(t, v) . \Sigma_{\bullet}^{<}(v, u) G^{A}\left(u, t^{\prime}\right) \\
t>t_{\mathrm{I}}, t^{\prime}>t_{\mathrm{I}}
\end{gathered}
$$

This equation does not depend on the time-partitioning method, but it illustrates the integration ranges for various terms, all within the $t>t_{\mathrm{I}}, t^{\prime}>t_{\mathrm{I}}$ quadrant of the double time plane. The propagators and their self-energies can be taken over from the host switch-on process; they are the same for both processes and we denote them simply $G^{R}, G^{A}, \Sigma^{R}, \Sigma^{A}$. The individual contributions to the self-energy $\Xi^{<}$corresponding to the process beginning at $t_{\mathrm{I}}$ can be expressed in terms of the quantities belonging to the host process. The result (obtained by time partitioning) is: 


$$
\begin{aligned}
& { }_{\mathrm{\alpha}} \Lambda^{<}\left(t_{\mathrm{I}}, u\right)=\mathrm{i} \int_{-\infty}^{t_{\mathrm{I}}} \mathrm{d} \bar{t}\left\{G^{R} \Sigma_{-\infty}^{<}+G_{-\infty}^{<} \Sigma^{A}\right\} \\
& \Lambda_{\mathrm{o}}^{<}\left(v, t_{\mathrm{I}}\right)=-\mathrm{i} \int_{-\infty}^{t_{\mathrm{I}}} \mathrm{d} \overline{\bar{t}}\left\{\Sigma_{-\infty}^{<} G^{A}+\Sigma^{R} G_{-\infty}^{<}\right\} \\
& \Sigma_{\bullet}^{<}(v, u)=\Sigma_{-\infty}^{<}(v, u) \\
& +\int_{-\infty}^{t_{\mathrm{I}}} \mathrm{d} \bar{t} \int_{-\infty}^{t_{\mathrm{I}}} \mathrm{d} \overline{\bar{t}}\left\{\Sigma^{R} G^{R} \Sigma_{-\infty}^{<}+\Sigma^{R} G_{-\infty}^{<} \Sigma^{A}+\Sigma_{-\infty}^{<} G^{A} \Sigma^{A}\right\}
\end{aligned}
$$

with

$$
\Sigma^{R} G^{R} \Sigma_{-\infty}^{<} \mapsto \Sigma^{R}(u, \bar{t}) G^{R}(\bar{t}, \overline{\bar{t}}) \Sigma_{-\infty}^{<}(\overline{\bar{t}}, v), \text { etc. }
$$

The external arguments $u, v$ are always greater than $t_{\mathrm{I}}$, while the integration variables $\bar{t}, \overline{\bar{t}} \leq t_{\mathrm{I}}$ belong entirely to the past. The condensed symbolic form of the integrations can be deciphered following the example in the last line of (19). It is apparent that in all integrands the arguments of the Green's functions never exceed $t_{\mathrm{I}}$, that is all propagations belong entirely to the past. By contrast, the self-energies have always one argument greater and the other one smaller than $t_{\mathrm{I}}$, in other words, they straddle the initial time, joining the past and the future in this sense.

We may elaborate on this last remark by showing explicitly the partitioning structure of the result. To this end, it is convenient to introduce the expressions (19) into Eq. (18). As a typical contribution to $G^{<}$we may take the first of the three integrals in the last line of (19):

$$
G^{<}=\cdots+\int_{t_{\mathrm{I}}}^{t} \mathrm{~d} v \int_{t_{\mathrm{I}}}^{t^{\prime}} \mathrm{d} u \int^{t_{\mathrm{I}}} \mathrm{d} \bar{t} \int_{\mathrm{I}}^{t_{\mathrm{I}}} \mathrm{d} \overline{\bar{t}} G^{R} \Sigma^{R} G^{R} \Sigma_{-\infty}^{<} G^{A}+\cdots
$$

All quantities in this expression, the Green's functions and the self-energies, belong to the host process, but they are partitioned, that is enter in blocks. For the Green's functions, the blocks are diagonal: either $t>$ $t_{\mathrm{I}}, t^{\prime}>t_{\mathrm{I}}$ (embedded process, propagation in the future with respect to the initial time), or $t<t_{\mathrm{I}}, t^{\prime}<t_{\mathrm{I}}$ (preparation process, propagation in the past with respect to the initial time). These diagonal blocks are coupled by the off-diagonal blocks of the self-energy with one argument greater and the other one smaller than $t_{\mathrm{I}}$, as pointed out above. This is typical for any partitioning method, only in our case it is applied on the Green's function understood as a matrix labelled by the two time variables.

These results have a conceptual and formal elegance, but do not appear as very useful in practical sense. The situation is different for a broad class of systems with a particular structure of the self-energy we are going to introduce now.

\subsection{Basic assumption about the time structure of the self-energy}

For many systems, it has been established or postulated that the self-energy as a function of two times is basically negligible, if the two time arguments are more apart from each other than some characteristic time. We have to distinguish the pair $\Sigma^{R}, \Sigma^{A}$ characterized by the so-called quasi-particle formation time $\tau_{\mathrm{Q}}$ and the less-self energy $\Sigma^{<}$whose characteristic time $\tau_{c}$ is often referred to as the collision duration time. In the double-time plane, the self-energy functions are concentrated to a strip around the time diagonal whose width is of the order of the relevant characteristic times, $\mathcal{O}\left(\tau_{Q}, \tau_{C}\right)$. We will denote this compound time by $\tau^{\star}$.

It follows that in the integrands entering Eq. (19) neither the outer times $u, v$ nor the integration variable times $\bar{t}, \overline{\bar{t}}$ can depart from $t_{\mathrm{I}}$ by more than $\tau^{\star}$. This has two important consequences for the problem of initial correlations - compare the related sketch in Fig. 1.

First, the Green's functions and the self-energies of the host process need to be known no deeper into the past than to times limited from below by $t_{\mathrm{I}}-\mathcal{O}\left(\tau_{Q}, \tau_{C}\right)$. This is a much weaker requirement than the knowledge of the preparation process over an endlessly extended past required in the general case. The selfenergy corrections (19) become a manageable and convenient means for incorporating the correlated initial conditions into the finite time NGF formalism.

Second, the initial correlations contribute to the selfenergy for times exceeding $t_{\mathrm{I}}$ by no more than about $\tau^{\star}$. This will get a deeper physical meaning shortly, as $\tau^{\star}$ will be identified with the Bogolyubov initial correlation decay time

$$
\tau^{\star} \equiv \tau_{\mathrm{B}}^{\star}
$$

in Sec. 5.1. This identification will permit us to use one or the other of the two $\tau^{\star}$ interchangeably in dependence on the context. 


\section{Towards transport equations}

The nature of a non-equilibrium transient evolution is decided by the hierarchy of characteristic times including the Bogolyubov correlation decay time $\tau_{\mathrm{B}}^{\star}$, the relaxation time $\tau$ closely related to the quasiparticle life-time, and a characteristic time of the external fields $T$. The ordering favoring the usual kinetic regime governed by a Boltzmann-like transport equation in extended systems, or by a master equation for small structures, is

$$
\tau_{\mathrm{B}}^{\star}=\tau^{\star} \ll \tau \ll T
$$

where we employ (20). The time scale of the transient is the largest and the whole $\mathbb{C}_{\text {I }}$ process evolves in three stages of a markedly different duration and physical nature: 1 . the decay of initial correlations between $t_{\mathrm{I}}$ and $t_{\mathrm{I}}+\tau^{\star} \ldots$ quasiparticle gas is forming, 2. the initial transient between $t_{\mathrm{I}}+\tau^{\star}$ and $t_{\mathrm{I}}+\tau \ldots$ memory of the initial state is being forgotten, and 3. the standard kinetic stage beyond $t_{\mathrm{I}}+\tau \ldots$ the quasiparticle distribution is gradually adjusting to the changing external field environment. The inequalities in (21) are of a different nature.

The condition $\tau^{\star} \ll \tau$ is a property of the interacting system itself and cannot be controlled from outside. The characteristic $\tau^{\star}$ time enters (21) only as a feature of the very early phase of the process. It is obvious from the definition of $\tau^{\star}$ in Sec. 4.4 that Eq. (21) is valid over the whole duration of the process. In fact, the $\tau^{\star} \ll \tau$ inequality is a precondition for use of the transport equations, even in their generalized quantum form. Otherwise, we would be bound to resort to solving the full NGF problem. In the following discussion, we take this condition for granted.

By contrast, the time scale of the external fields is among the control parameters. If the field variation will be slow, as required by Eq. (21), a standard transport formalism can be developed and applied. This includes, in particular, the case of steady transport as a response to suddenly applied constant external fields. At the opposite side of the time scale for external fields is their sudden, jump-like change. This case may be treated as if a renewed process started at the instant of the jump from an initial condition prepared during the preceding evolution.

Finally, a situation of particular interest will in many cases be specified by a relaxed Eq. (21),

$$
\tau^{\star} \ll \tau \sim T
$$

which defines the domain of quantum transport /generalized master equations. The discussion in the remaining sub-parts of this section will be based on Eq. (22). As will be apparent, the three stages of evolution of a transient starting at $t_{\mathrm{I}}$ may be distinguished under these more general conditions as well.

\subsection{Decay of initial correlations}

Right after the start of the transient, the evolution is fully influenced by the correlated initial state and it requires a complete quantum dynamical description. In terms of the cyclic scheme (16), both the precursor equation and the reconstruction equations must be used in their exact form, Eq. (11) and Eq. (15), respectively. The decay of initial correlations is assumed to be fast by Eq. (22). Using the reasoning given in Sec. 4.4 , it may be concluded that after elapse of a time of the order of $\tau^{\star} \sim \mathcal{O}\left(\tau_{Q}, \tau_{C}\right)$, the precursor equation will simplify, because all corrections for initial correlations will vanish and we get

$$
\begin{aligned}
G_{0}^{-1} G^{<}-G^{<} G_{0}^{-1}= & \Sigma^{R} G^{<}-G^{<} \Sigma^{A} \\
& -G^{R} \Sigma_{-\infty}^{<}+\Sigma_{-\infty}^{<} G^{A}
\end{aligned}
$$

A similar simplification takes place also in the reconstruction equations, as will be discussed in detail in the next section, so that the Bogolyubov conjecture is substantiated for the class of systems whose self-energy may be characterized by a finite characteristic time $\tau^{\star}$ which thus becomes the interpretation of the correlation decay time. In particular, we identify $\tau^{\star} \equiv \tau_{\mathrm{B}}^{\star}$, as anticipated already in Eq. (20).

While the precursor transport equation, and, consequently, any transport equation obtained as its approximation, is free of memory of the initial conditions after the initial correlations have decayed, the solution of the equation itself keeps the memory of the state, from which it stems. This is seen from the Dyson equation (18) and the explicit expressions for self-energies in Eq. (19). The complete solution consists of two parts, the coherent initial transient which decays at a rate given by the quasiparticle relaxation time $\tau$ and a term which tends at the same rate to the long time solution 


$$
\begin{aligned}
& \rho(t)=-\mathrm{i} G^{<}(t, t) \\
& \stackrel{t \rightarrow \infty}{\longrightarrow}-\mathrm{i} \int_{t_{\mathrm{I}}}^{t} \mathrm{~d} v \int_{t_{\mathrm{I}}}^{t} \mathrm{~d} u G^{R}(t, v) \Sigma_{-\infty}^{<}(v, u) G^{A}(u, t)
\end{aligned}
$$

identical with the solution of the host process: Beyond a time $\sim t_{\mathrm{I}}+2 \tau$ the lower integration limits may be replaced by $-\infty$ and the initial transient does not enter any more.

\subsection{Reduction of the reconstruction equations}

The reconstruction equations (15) also depend on the correlated initial condition, which enters through the self-energy $\Xi^{<}$again. This is important only for the earliest times, however. Once the external times exceed the initial time by more than $\sim 2 \tau^{\star}$, say $t \geq$ $t^{\prime} \geq t_{\mathrm{I}}+2 \tau^{\star}$, the initial correlations cease to contribute to Eq. (15): it then holds $\overline{\bar{t}}>t_{\mathrm{I}}+2 \tau^{\star}$ and this means that all initial-correlation terms contributing to $\Xi^{<}$ vanish. The equation then reduces to a reconstruction equation without initial correlations. All dependence on the initial correlations is but implicit, through the free term containing $\rho$, which accumulates the memory of the past starting from $t_{\mathrm{I}}$. This points to the central position of $\rho$ in the cyclic process (16) again.

It is interesting to trace down formal origins of the difference between the reconstruction equations and the full Dyson equation. The last integral in (15) involving the initial conditions is similar to its analogue in (18). The integration area is different, however. While it is a whole rectangle with the corners $\left(t_{\mathrm{I}}, t_{\mathrm{I}}\right)$ and $\left(t, t^{\prime}\right)$ in the Dyson equation, it only extends over its upper part with the lower left corner shifted to $\left(t^{\prime}, t_{\mathrm{I}}\right)$ for the reconstruction equation. As a consequence, as soon as $t^{\prime}>t_{\mathrm{I}}+2 \tau^{\star}$, the initial-correlation term vanishes in the reconstruction equation (15), while it remains in the Dyson equation (18) - in agreement with the previous observation that the characteristic time for the full Dyson equation is $\tau$.

\subsection{Summary to the reconstruction cycle (16)}

With the above results, it is possible to illuminate the technical alternative for generating full $G^{<}$correlation function as offered by the Reconstruction theorem underlying the cyclic scheme of Eq. (16). We may contrast this alternative with a direct use of the Dyson equation (18). The Dyson equation has the form of an explicit formula for $G^{<}$once the self-energy $\Xi^{<}$is known and the initial conditions are bound to enter it explicitly. By contrast, the reconstruction equations are true integral equations, incorporating the initial conditions in an oblique manner and imposing them on the solution $G^{<}$successively with an increasing time. The reconstruction equations are not stand-alone objects, as they require the density matrix $\rho(t)$ as the input, which is provided by the other constitutive part of the iterative solution cycle, the precursor transport equation in an exact formulation, or its QTE clone in an approximate version. In turn, $G^{<}$from the reconstruction equations serves as an input for the transport equation.

The initial conditions enter this cyclic process both in the reconstruction equations and in the precursor transport equation explicitly, but in both cases only within the earliest period of time, $t<t_{\mathrm{I}}+\mathcal{O}\left(\tau^{\star}\right)$, which we called above the first stage of the transient, or the decay of initial correlations. Clearly, the reconstruction cycle offers no advantage within this stage and some other method may be preferable.

During the ensuing second and third stages of the transient development, the initial conditions do not intervene in the equations of the reconstruction cycle. Altogether, a formalism for treating transient processes results, which is different for the first stage, and for the rest of the process: All initial conditions are built in during the initial period, subsequently they are propagated by the dynamics of the process at later times. The distinction between the second and the third stages is not sharp: they are treated in the same way, only the quantities involved, $\rho$ and $G^{<}$, include the initial transient during the second stage, while they are insensitive to the initial conditions at the third, kinetic stage.

There are two consequences. Firstly, the differing roles of the initial conditions in the Dyson equation and the QTE context become harmonized. Secondly, the outlined QTE scheme fully corroborates the Bogolyubov conjecture described in Section 3.

\section{QTE and non-equilibrium quasiparticles}

Finally, we arrive at the task of an approximate direct construction of a quantum transport equation ap- 
propriate for the second and third stages of the evolution. In other words, of shortcutting the

$\{$ reconstruction equations $\rightleftharpoons$ precursor $\mathrm{QTE}\}$ cycle (16). By now, we are in the position to discuss, how the finite time initial conditions affect the possibilities of this step.

6.1. Quantum transport equation by GKBA: multiplicative decoupling - the point of departure

The most widely used method has been based on the GKBA (13). This is an approximation neglecting the integral terms in the reconstruction equation (15). This also includes omitting the initial correlations. The GKBA itself is, as pointed out is Sec. 3.2, too coarse to take them into account. Once the running time of the process has already entered the second stage of evolution, GKBA may be combined with the reduced precursor QTE (23), and it yields a closed transport equation, which is free of explicit initial correlations in the generalized scattering terms. Obviously, no QTE theory may have the ambition to provide a detailed description of short time correlations, as this is precluded by the Bogolyubov principle; the QTE is of an asymptotic long time nature.

GKBA has not only been widely used, but it also was extremely successful in practical numerical calculations. This is somewhat surprising from a theoretical point of view, as the Ansatz basically amounts to a factorization

$$
\left.G^{<}\left(t, t^{\prime}\right) \stackrel{?}{=} \mathrm{i} G^{R}\left(t, t^{\prime \prime}\right) G^{<}\left(t^{\prime \prime}, t^{\prime}\right)\right|_{t^{\prime \prime}=t^{\prime}}
$$

which has no formal justification. This difficulty got amplified, when GKBA was derived in an alternative way $[10,11,28,49]$ and the GKBA factorization has been linked with a similar multiplicative rule for propagators, the so-called semi-group composition rule. The latter reads, for a fixed $t^{\prime \prime}$,

$$
\begin{array}{ll}
G^{R}\left(t, t^{\prime}\right)=G^{R}\left(t, t^{\prime}\right), & t^{\prime \prime} \geq t \geq t^{\prime} \\
G^{R}\left(t, t^{\prime}\right)=\mathrm{i} G^{R}\left(t, t^{\prime \prime}\right) G^{R}\left(t^{\prime \prime}, t^{\prime}\right), & t \geq t^{\prime \prime} \geq t^{\prime}
\end{array}
$$

for a retarded propagator; similarly for the advanced function. While the GKBA factorization is not very transparent, it is easy to see that the multiplicative rule cannot really be sustained for the propagator. The error will be the larger, the stronger will be the correlations in the system: the propagator will start from a decay of correlations during the early times up to $\sim \tau^{\star}$ after the injection. This is called the formation process for propagators - and the propagator will change fast during it. Such behavior of $G^{R}\left(t, t^{\prime \prime}\right)$ will bring about an unphysical steep decline on the factorized l.h.s. of (26). In equilibrium, the relative magnitude of the jump is $|1-Z|$ with $Z$ being the renormalization constant of the quasiparticle (residuum of the quasiparticle pole) .

It has been pointed out in $[10,28]$, that Eq. (26) is a constitutive functional equation for non-equilibrium quasiparticle propagators extending out of equilibrium the functional equation $\exp (a+b)=\exp (a) \times \exp (b)$ governing the equilibrium quasiparticle propagators. This suggested that also the Ansatz factorization should invoke rather the quasiparticle propagators, that is, the GKBA should be modified to a "QKBA", the Quasiparticle Kadanoff-Baym Ansatz. Such procedure is barely uniquely defined, but we will outline in more detail one possibility. A natural guess for QKBA (written in resemblance with (25)) is

$$
\left.G^{<}\left(t, t^{\prime}\right) \stackrel{?}{=} \mathrm{i}_{Q}^{R}\left(t, t^{\prime \prime}\right) G^{<}\left(t^{\prime \prime}, t^{\prime}\right)\right|_{t^{\prime \prime}=t^{\prime}}
$$

where $G_{Q}^{R}$ denotes a quasi-particle propagator - unspecified as yet. In the two sub-sections to follow we will give Eq. (27) some interpretation and justification.

\subsection{Non-equilibrium quasi-particles}

First, the non-equilibrium quasiparticles are defined. We use a purely formal definition of a quasi-particle, whose only indication is a specific time structure of its propagator. In equilibrium, as reminded above, the quasi-particles are slowly exponentially decaying resonances. Beyond the adiabatic regime, where the spectral representation common in the Fermi liquid theory is not available, the non-stationary quasi-particles have to be described entirely in terms of properties of time-dependent quantities. We postulate that a quasiparticle formation time $\tau_{\mathrm{Q}}$ exists such that the following pair of functional equations holds for all $t$ and $t^{\prime}$ within a certain time domain and replaces there the heuristically proposed Eq. (26):

$$
\begin{aligned}
G^{R}\left(t, t^{\prime}\right) & =\mathrm{i} G_{Q}^{R}\left(t, t^{\prime \prime}\right) G^{R}\left(t^{\prime \prime}, t^{\prime}\right), \\
G_{Q}^{R}\left(t, t^{\prime}\right) & =\mathrm{i} G_{Q}^{R}\left(t, t^{\prime \prime}\right) G_{Q}^{R}\left(t^{\prime \prime}, t^{\prime}\right), \\
& t \geq t^{\prime \prime}>t^{\prime}+\mathcal{O}\left(\tau_{\mathrm{Q}}\right)>t^{\prime}
\end{aligned}
$$


The first equation (28) may be termed the Quasiparticle Composition Rule (QCR). It introduces a new quasiparticle propagator $G_{Q}^{R}$ by building it from the true retarded Green's function. The second functional equation (29) postulates for the quasiparticles the behavior of metastable independent particles: they should obey the Semigroup Composition Rule proper.

This second rule alone defines a whole class $\mathcal{W}$ of quasi-particle propagators $G_{W}^{R}(\ldots W$ tribute to Wigner and Weisskopf). Equivalently, this class is formed by propagators possessing a time-local selfenergy. Each member of the class is then specified by its optical potential $\sigma_{\mathrm{w}}(t)$. This correspondence can be given in two equivalent formulations,

$$
\begin{aligned}
& \text { a. } \Sigma_{W}^{R} \text { time diagonal: } \Sigma_{W}^{R}\left(t, t^{\prime}\right)=\sigma_{\mathrm{w}}(t) \delta\left(t-t^{\prime}+\mathrm{i} 0\right) \\
& \text { b. } G_{W}^{R}\left(t, t^{\prime}\right)=-\mathrm{i} T \mathrm{e}^{-\mathrm{i} \int_{t^{\prime}}^{t} \mathrm{~d} u\left\{H_{\mathrm{MF}}(u)+\sigma_{\mathrm{w}}(u)\right\}}
\end{aligned}
$$

The self-energy in a. is infinitesimally retarded, $T$ appearing in $\mathbf{b}$. is the time-ordering operator and $H_{\mathrm{MF}}$ is the Hermitian time-local mean-field Hamiltonian.

The non-equilibrium quasi-particle propagator defined in this way has the desired physical meaning. It is formally similar to the mean-field propagator, which describes a unitary evolution of independent effective particles moving in the average external and inner fields. In $G_{W}^{R}$, the optical potential $\sigma_{\mathrm{W}}$ enters in addition. The evolution need not be unitary any more, yet it is governed by an effective single particle Hamiltonian, albeit typically non-Hermitian. Hence, we may speak of a flight of independent metastable quasi-particles.

It remains to find the specific optical potential $\sigma_{\mathrm{Q}}$ belonging to a prescribed true propagator. The input is, of course, the propagator itself and/or the related self-energy. For fixed $t^{\prime}$ and $t^{\prime \prime}$, the QCR (28) for a running upper time $t$ is equivalent with the condition

$$
\int \mathrm{d} \bar{t} \Sigma^{R}(t, \bar{t}) G^{R}\left(\bar{t}, t^{\prime}\right) \approx \sigma_{\mathrm{Q}}(t) G^{R}\left(t, t^{\prime}\right)
$$

independently of $t^{\prime}$ (and $t^{\prime \prime}$ ). This equation may serve for obtaining the optical potential.

But will a given self-energy produce the quasiparticle optical potential $\sigma_{\mathrm{Q}}$ at all? The answer is positive for equilibrium. In a transient situation, the equality sign in (31) is but approximate, as also indicated. Heuristically, we expect a good approximant to $\sigma_{\mathrm{Q}}$ to exist in the case that $\tau_{\mathrm{Q}}$ is an atom of time: if the GF will change smoothly, the integral in (31) will act as a short time sampling of the Green's function insensitive to its general structure including its $t^{\prime}$ dependence. This will work, if the system will obey the following

QUASI-PARTICLE EXISTENCE CONDITIONS

$\diamond$ Relaxation in the system should be slow: $\tau \gg \tau_{\mathrm{Q}}$

$\diamond$ The external fields should be smooth on the $\tau_{\mathrm{Q}}$ scale

$\diamond$ The times in question are restricted by

$$
t>\breve{t} \equiv \inf _{\mathrm{QCR}} t^{\prime \prime} \approx t^{\prime}+\mathcal{O}\left(\tau_{\mathrm{Q}}\right)>t^{\prime}
$$

These conditions specify how far we stretch the quasiparticle concept beyond the adiabatic limit (21); the first two conditions together are equivalent with the QTE conditions (22) and give it a deeper meaning. This corroborates our assertion that the QTE regime hinges upon the use of non-equilibrium quasi-particles. As concerns the individual conditions, the first one is crucial. If the relaxation in the system is exceedingly fast, the quasi-particles are bound to die out before getting formed. The second condition puts an upper limit at rate of change of the external fields. The quasiparticles under transient external conditions are forced to steadily readjust to the time-variable environment and they need that the environment appear as steady over the formation time. Finally, the third condition will be violated, if the splitting time will not obey the last inequality (32), so that $\breve{t}>t^{\prime \prime}>t^{\prime}$, the system will be in the period of quasi-particle formation and the average (31) will not be possible.

For the actual construction of an optimized approximate $\sigma_{\mathrm{Q}}$ beyond the adiabatic limit, the above argument has to be refined. For that, it is advantageous to develop the so-called phase equation [28] reminiscent somewhat of the Calogero equations of the scattering theory. This will not be analyzed here.

\subsection{Quasi-particle reconstruction equations}

With the non-equilibrium quasi-particle definition at hand, we will be able to develop and discuss an analogue of the reconstruction equations (15) involving the quasi-particle propagation instead of the true one.

To get a better understanding of the related modifications, it will be convenient to first complement 
Eq. (15) by its analogue valid for propagators, the renormalized semi-group rule $[10,11]$ :

$$
\begin{gathered}
G^{R}\left(t, t^{\prime}\right)=\mathrm{i} G^{R}\left(t, t^{\prime \prime}\right) G^{R}\left(t^{\prime \prime}, t^{\prime}\right)+ \\
\int_{t^{\prime \prime}}^{t} \mathrm{~d} \bar{t} \int_{t^{\prime}}^{t^{\prime \prime}} \mathrm{d} \overline{\bar{t}} G^{R}(t, \bar{t}) \Sigma^{R}(\bar{t}, \overline{\bar{t}}) G^{R}\left(\overline{\bar{t}}, t^{\prime}\right) \\
\quad t \geq t^{\prime \prime} \geq t^{\prime}
\end{gathered}
$$

We have altogether two pairs of relations. Each pair consists of an exact integral equation accompanied by its zeroth iteration approximant - entering as a source term. Specifically, Eq. (33) is a renormalized semigroup rule (26) for $G^{R}$. Similarly, the source term of the reconstruction equation (15) for $G^{<}$coincides with the GKBA expression (25).

The integrals in the exact equations augment the source expressions as vertex corrections. Their physical meaning is seen from peculiar integration limits: they link the past and the future with respect to the splitting time $t^{\prime \prime}$ and blur the point-like dividing line between both time domains inherent to the sharp factorization of the sources. The magnitude of the vertices decides about the quality of the simple factorizations in the source terms.

There is but one vertex correction in Eq. (33). In (15), the two integrals represent two vertex corrections of a rather different nature. The first one describes a single particle ("polaron") renormalization on the outchannel. The second one has an irreducible character and captures the two-particle correlations which surpass the Ansatz level.

It is not difficult to obtain the quasi-particle counterparts of Eqs. (33) and (15). Quite formally, we start by relating any quasi-particle propagator $G_{W}^{R} \in \mathcal{W}$ and the true propagator $G^{R}$ by the ("relative") Dyson equation

$$
\begin{aligned}
& G^{R}\left(t, t^{\prime}\right)=G_{W}^{R}\left(t, t^{\prime}\right)+ \\
& \int_{t^{\prime}}^{t} \mathrm{~d} \bar{t} \int_{t^{\prime}}^{\bar{t}} \mathrm{~d} \overline{\bar{t}} G_{W}^{R}(t, \bar{t})\left\{\Sigma^{R}-\Sigma_{W}^{R}\right\}(\bar{t}, \overline{\bar{t}}) G^{R}\left(\overline{\bar{t}}, t^{\prime}\right)
\end{aligned}
$$

By this, the true propagator is decomposed into the "free" propagator and the self-energy correction both of which are functionals of the variable $\sigma_{\mathrm{w}}(t)$.

$\diamond$ For any intermediate time $t^{\prime \prime}$, this Dyson equation can be rearranged to the renormalized QCR

$$
\begin{aligned}
& G^{R}\left(t, t^{\prime}\right)=\mathrm{i} G_{W}^{R}\left(t, t^{\prime \prime}\right) G^{R}\left(t^{\prime \prime}, t^{\prime}\right)+ \\
& \int_{t^{\prime \prime}}^{t} \mathrm{~d} \bar{t} \int_{t^{\prime}}^{\bar{t}} \mathrm{~d} \overline{\bar{t}} G_{W}^{R}(t, \bar{t})\left\{\Sigma^{R}-\Sigma_{W}^{R}\right\}(\bar{t}, \overline{\bar{t}}) G^{R}\left(\overline{\bar{t}}, t^{\prime}\right)
\end{aligned}
$$

On this transformation, the free term $G_{W}^{R}$ is replaced by the operator product $\mathrm{i}_{W}^{R} G^{R}$ extending the desired form (28) to any member of the $\mathcal{W}$ class. In the vertex correction, the self-energy is replaced by the difference $\Sigma^{R}-\Sigma_{W}^{R}$ and the outer integration range is narrowed upwards to $t^{\prime \prime}<\bar{t}<t$. The new integration limits guarantee that only $\sigma_{\mathrm{W}}(t)$ for $t>t^{\prime \prime}$ enters (35).

$\diamond$ The "quasi-particle reconstruction equation" for $t>$ $t^{\prime}$ is obtained from Eq. (15), if the retarded propagator is rewritten with the use of Eqs. (34), (35). After minor manipulations, the result is

$$
\begin{aligned}
& G^{<}\left(t, t^{\prime}\right)=-G_{W}^{R}\left(t, t^{\prime}\right) \rho\left(t^{\prime}\right) \\
&+\int_{t^{\prime}}^{t} \mathrm{~d} \bar{t} \int_{t_{\mathrm{I}}}^{\bar{t}} \mathrm{~d} \overline{\bar{t}} G_{W}^{R}(t, \bar{t})\left\{\Sigma^{R}-\Sigma_{W}^{R}\right\}(\bar{t}, \overline{\bar{t}}) G^{<}\left(\overline{\bar{t}}, t^{\prime}\right) \\
& \quad+\int_{t^{\prime}}^{t} \mathrm{~d} \bar{t} \int_{t_{\mathrm{I}}}^{t^{\prime}} \mathrm{d} \overline{\bar{t}} G^{R}(t, \bar{t}) \Xi^{<}(\bar{t}, \overline{\bar{t}}) G^{A}\left(\overline{\bar{t}}, t^{\prime}\right)
\end{aligned}
$$

The source term, with the true propagator $G^{R}$ replaced by $G_{W}^{R}$, is identical with the $t>t^{\prime}$ half of QKBA shown in $(27)$, but with the optical potential $\sigma_{\mathrm{w}}$ not specified so far:

$$
G^{<}=-G_{W}^{R} \rho+\rho G_{W}^{A} .
$$

Naturally, $G_{W}^{R}\left(\Sigma^{R}-\Sigma_{W}^{R}\right)$ replaces $G^{R} \Sigma^{R}$ in the "polaron" term. By contrast, the irreducible vertex correction is the same in (36) and in (15), and it will be preserved and its proper inclusion will be necessary under any renormalization of the propagators. Notice that while the initial-correlation terms are formally included in the self-energy $\Xi^{<}$, they are inactive for times obeying the conditions (32).

\subsection{Resulting quasi-particle QTE scheme}

The advantage of the quasiparticle renormalization in (35), (36) stems from the replacement $\Sigma^{R} \rightarrow \Sigma^{R}-\Sigma_{W}^{R}$ in the "polaron" part of the vertex. If the quasi-particle existence conditions (page 15) are satisfied, then an optimization procedure, based preferably on the phase equation as outlined in Sec. 6.2 , yields the quasi-particle optical potential $\sigma_{\mathrm{Q}}$ for 
which the quasi-particle propagators are well defined and obey the simplified equations (28), (29) and their analogue for $G^{A}, G_{Q}^{A}$. The reconstruction equation for the particle correlation function also simplifies to

$$
\begin{aligned}
& G^{<}\left(t, t^{\prime}\right)=-G_{Q}^{R}\left(t, t^{\prime}\right) \rho\left(t^{\prime}\right)+\rho(t) G_{Q}^{A}\left(t, t^{\prime}\right) \\
& \left\{\int_{t^{\prime}}^{t} \mathrm{~d} \bar{t} \int_{t_{\mathrm{I}}}^{t^{\prime}} \mathrm{d} \overline{\bar{t}}+\int_{t}^{t^{\prime}} \mathrm{d} \overline{\bar{t}} \int_{t_{\mathrm{I}}}^{t} \mathrm{~d} \bar{t}\right\} G^{R}(t, \bar{t}) \Xi^{<}(\bar{t}, \overline{\bar{t}}) G^{A}\left(\overline{\bar{t}}, t^{\prime}\right)
\end{aligned}
$$

where the irreducible vertex persists. This term would be neglected on the level of accuracy of the usual GKBA and we would simply use the QKBA instead in the cyclic process

$\{$ reconstruction equations $\stackrel{\text { QKBA }}{\rightleftharpoons}$ precursor QTE $\}$ of Eq. (16). Just as in the case of the standard KBA or GKBA, the cycle can be contracted to a single quantum transport equation.

QTE is effectively a single-time (integro-)differential equation. The depth of the integrals constituting the generalized scattering terms is $\tau$. The single-time nature of the equation is connected with the other time scale: the atom of time is $\sim \tau^{\star}$, much shorter than $\tau$, and the effective single-time quantities at a given time result from a complicated sampling over the relevant time window. The same is true about the underlying quasi-particles themselves. They cannot exist out of the context of the particular system in motion; the sampling procedure for the quasi-particle optical potential is given by Eq. (32).

It remains to clarify the initial conditions for the QTE. To this, we return to the introduction of Sec. 5, where we discussed the three stages of evolution of a transient process. The shortest first stage between $t_{\mathrm{I}}$ and $t_{\mathrm{I}}+\mathcal{O}\left(\tau^{\star}\right)$ encompasses the decay of initial correlations during which the quasiparticle gas is forming. The transport equation cannot be used, it comes into power only with the onset of the second stage of evolution. In the second stage - the initial transient- the system keeps memory of the initial conditions. For the transport equation, the initial conditions are fully contained in the "initial value" of $\rho$. We use quotes here, because the starting value for $\rho$ is given by the evolution over the initial period lasting up to $t_{\mathrm{I}}+\tau_{\mathrm{SAT}}, \tau_{\mathrm{SAT}}=\mathcal{O}\left(\tau^{\star}\right)$. The precise length $\tau_{\mathrm{SAT}}$ of this period is not critical, as at the end of the first stage, the evolution saturates to a moderate rate $\sim \max \left(\tau^{-1}, T^{-1}\right)$ characterizing the rest of the whole process - see Eq. (22). The effective initial condition must be obtained from a complete solution for the process, as explicitly given by the Dyson equation (4), (18), and it includes the effect of initial correlations as it comes from the $\Lambda$ 's sampled over the same time interval. Because the time resolution for the transport equation, the "atom of time", is also $\sim \tau^{\star}$, we are entitled to make the identification

$$
\rho\left(t_{\mathrm{I}}\right)=-\mathrm{i} G^{<}\left(t_{\mathrm{I}}+\tau_{\mathrm{SAT}}, t_{\mathrm{I}}+\tau_{\mathrm{SAT}}\right)
$$

Similarly, the generalized collision terms have as their lower integration limit just $t_{\mathrm{I}}$ during the initial transient. Only in the full kinetic stage, the precise value of this lower limit becomes unimportant, as it becomes gliding at a value $t-\mathcal{O}(\tau)$.

Returning to the choice of Ansatzes, we may improve on the plain QKBA and take account of the vertex corrections by iterating the reconstruction equation; this would capture two types of corrections, the irreducible vertex determining the effect of collisions on the evolution of $G^{<}$, and the correction for the residual error of the approximate minimization of the "polaron" term. The result would be a renormalized "RQKBA".

The reconstruction cycle could be shortened to a single quantum transport equation again: there is a uniform procedure by which any Ansatz will yield a quantum transport equation. The resulting equations will differ "merely" in the details of the generalized scattering terms. Just these details are decisive for the physical consistency and quality of each of the equations. The expectation is that their reliability will increase in the sequence reflecting the degree of self-consistency and improved renormalization, $\mathrm{GKBA} \rightarrow \mathrm{QKBA} \rightarrow \mathrm{RQKBA}$

\subsection{Judging the quality of Ansatzes}

We have to begin by asking, how the quality of an Ansatz and of the associated QTE can be judged. It should be stressed that already the original GKBA possesses a number of properties which make it consistent with the general NGF scheme [10]. In the first place, it has the causal structure, as given by Eq. (13), that is, it automatically keeps the causal order of the factors $\rho$, $G^{R}$ and $G^{A}$. The time argument of $\rho$ is, in both terms, always the smaller of the two time arguments $\left(t, t^{\prime}\right)$ of the correlation function $G^{<}$. The reduced density matrix $\rho$ represents thus a floating initial condition, while 
the propagators $G^{R, A}$ describe evolution of the system from the initial condition to a later time. Another key property of the Ansatz is that it is a leading term of an iterative procedure for the reconstruction equation which cannot be directly linked with a perturbative expansion in terms of particle-particle interaction, so that GKBA is non-perturbative.

The same properties are found also for the other two Ansatzes above; in fact, they are characteristic of a wide family of causal Ansatzes having the general form

$$
G^{<} \approx \rho G_{\bullet}^{A}-G_{\bullet}^{R} \rho
$$

where $G_{\bullet}^{R, A}$ are specific/optional choices of propagators, like $G^{R, A}$ proper for GKBA, or $G_{Q}^{R, A}$ for QKBA; a more general example is provided by (37). As concerns the general expression (40), all renormalizations are condensed in the replacement $G^{R, A} \mapsto G_{\bullet}^{R, A}$, while the distribution function for true particles is kept unchanged. (Alternatively, we could transfer at least a part of the renormalization on the distribution function, transforming it to a generalized quasi-particle distribution.)

We see that it would be hard to distinguish between various Ansatzes by their general qualitative properties. On the other hand, all these qualitative properties do not guarantee that an Ansatz will be quantitatively satisfactory. All this points to the importance of quantitative criteria, notably conserving properties of the approximations involved. Particle number conservation is the simplest among them and the related criterion is the Transport Ward identity[29-31,7,32,33]. It is necessary, of course, to begin by extending this identity to non-equilibrium and to processes starting from correlated initial conditions. This will be analyzed in the coming section.

\section{Ward identities out of equilibrium}

The Ward identities out of equilibrium were introduced in [49]. They extend the purpose and the scope of the equilibrium Ward identities to arbitrary nonequilibria including fast transients. Here, we will extend the considerations of [49] to processes starting from a correlated initial condition at a finite initial time. The original aim was to establish rules constraining the choice of diagrams for processes of different order and, hence contribute to construction of reliable perturbation schemes. This facette can be extended also out of equilibrium, but we will not dwell on it here.

The Ward identities, physically speaking, allow to express, in some special cases, two-particle characteristics of the system by means of single particle quantities. This offers a non-trivial possibility to move "upwards" in the hierarchy of multiparticle complexes, contrary to the usual tracing procedure permitting to move downwards between the one and two particle level.

There is a wide class of Ward identities, but we will concentrate on just one case, namely the Ward identities for the transport vertex. They are directly related to our subject-matter. Close to equilibrium, where this language is admissible, they may be said to provide a consistency check between approximations for spectral and transport properties. Out of equilibrium, we may use the spectral language but conditionally and the whole problem has to be reformulated entirely in the time domain. A distinction between single-particle propagators and the response of the system as a whole to an external field remains preserved. In homogeneous systems (like in the original Ward's treatment of QED), it makes sense to introduce, in addition to the Ward identity for the scalar vertex related to the particle number conservation, also similar identities for the momentum vertices. Here, we have in mind mostly application of these techniques to small strongly inhomogeneous systems. Even for them, the scalar Ward identity remains universally valid.

\subsection{Global gauge symmetry}

For an arbitrary external time-local disturbance $U(t)$, the field dependent GF and self-energy satisfy

$$
\begin{gathered}
\mathbf{G}_{\mathcal{U}}=\mathbf{G}_{0 \mathcal{U}}+\mathbf{G}_{0 \mathcal{U}} \boldsymbol{\Sigma}_{\mathcal{U}} \mathbf{G}_{\mathcal{U}} \\
\mathbf{G}_{0 \mathcal{U}}^{-1}=\mathbf{G}_{0}^{-1}-\mathbf{U} ; \quad \mathbf{U}\left(t, t^{\prime}\right)=\delta\left(t-t^{\prime}\right)\left\|\begin{array}{cc}
U(t) & 0 \\
0 & U(t)
\end{array}\right\|
\end{gathered}
$$

The vertex corrections appear, in an integral form, as $\delta_{\mathcal{U}} \boldsymbol{\Sigma}$ in the linear response of the one-electron GF to a small variation $\delta \mathbf{U}$ of the external field. By Eq. (41):

$$
\delta_{\mathcal{U}} \mathbf{G}=\mathbf{G} \delta_{\mathcal{U}}\left[-\mathbf{G}^{-1}\right] \mathbf{G}=\mathbf{G}\left[\delta \mathbf{U}+\delta_{\mathcal{U}} \boldsymbol{\Sigma}\right] \mathbf{G}
$$

The gauge invariance of the first kind (in other words, the global $U(1)$ symmetry) is invoked as follows: a time variable shift $\mathcal{U}(t)$ is added to the one- 
particle energy in the free GF. This is obtained by a particular external disturbance,

$$
\mathbf{G}_{0 \mathcal{U}}^{-1}=\mathbf{G}_{0}^{-1}-\mathcal{U}(t) \times \delta\left(t-t^{\prime}\right) \mathbf{1}, \quad \mathbf{1} \equiv\left\|\begin{array}{ll}
I & 0 \\
0 & I
\end{array}\right\|
$$

with the spatially homogeneous potential energy $\mathcal{U}(t)$ being an arbitrary real function of time, but not of canonical variables, and $I$ the operator unity.

The field dependence of the Dyson equation is then expressed by explicit relations

$$
\mathbf{X}_{\mathcal{U}}\left(t, t^{\prime}\right)=\mathbf{X}\left(t, t^{\prime}\right) \cdot \exp \left(-\mathrm{i} \int_{t^{\prime}}^{t} \mathrm{~d} \tau \mathcal{U}(\tau)\right)
$$

where $\mathbf{X}=\mathbf{G}, \mathbf{G}_{0}, \boldsymbol{\Sigma}$. For $\mathbf{G}, \mathbf{G}_{0}$, (44) follows from definition and the Fermion number conservation. The self-energy $\boldsymbol{\Sigma}$ must transform according to Eq. (44) to keep the Dyson equation (gauge) invariant. This nontrivial requirement leads to the Ward identity.

\subsection{Ward identity with initial condition}

From the gauge transformation (44), the vertex correction $\delta_{\mathcal{U}} \boldsymbol{\Sigma}$ follows as

$$
\delta_{\mathcal{U}} \boldsymbol{\Sigma}\left(t, t^{\prime}\right)=\boldsymbol{\Sigma}\left(t, t^{\prime}\right) \cdot\left(-\mathrm{i} \int_{t^{\prime}}^{t} \mathrm{~d} \tau \delta \mathcal{U}(\tau)\right)
$$

We define an operator vertex correction having three time terminals. By Eq. (45), it has an explicit form expressing the three-point vertex $\boldsymbol{\Delta}$ in term of a twopoint self-energy $\boldsymbol{\Sigma}$. This is the non-equilibrium Ward identity we sought for:

$$
\begin{aligned}
& \boldsymbol{\Delta}\left(t, t^{\prime} ; t_{\times}\right) \equiv \frac{\delta}{\delta \mathcal{U}\left(t_{\times}\right)} \boldsymbol{\Sigma}\left(t, t^{\prime}\right)=-\mathrm{i} \boldsymbol{\Sigma}\left(t, t^{\prime}\right) \cdot \eta\left(t, t^{\prime} ; t_{\times}\right) \\
& \eta\left(t, t^{\prime} ; t_{\times}\right)=\vartheta\left(t-t_{\times}\right) \vartheta\left(t_{\times}-t^{\prime}\right)-\vartheta\left(t^{\prime}-t_{\times}\right) \vartheta\left(t_{\times}-t\right)
\end{aligned}
$$

This result is formally identical with the nonequilibrium Ward Identity derived in [49] for the case of Keldysh uncorrelated initial condition. Presently, however, the self-energy, and, hence, the related vertex, have additional singular parts in the less-component. By Eq. (10) and (46),

$$
\begin{gathered}
\Delta^{<}\left(t, t^{\prime} ; t_{\times}\right)=._{\circ} \Delta_{\circ}^{<}+{ }_{\circ} \Delta_{\bullet}^{<}+._{\bullet}^{<} \\
\Delta_{\circ}^{<}\left(t, t^{\prime} ; t_{\times}\right)=-\mathrm{i} \Sigma_{\circ}^{<}\left(t, t_{\mathrm{I}}\right) \vartheta\left(t-t_{\times}\right) \\
\Delta_{\bullet}^{<}\left(t, t^{\prime} ; t_{\times}\right)=+\mathrm{i}_{\circ} \Sigma_{\bullet}^{<}\left(t_{\mathrm{I}}, t^{\prime}\right) \vartheta\left(t^{\prime}-t_{\times}\right) \\
\boldsymbol{.}_{\bullet}^{<}\left(t, t^{\prime} ; t_{\times}\right)=-\mathrm{i} \Sigma_{\bullet}^{<}\left(t, t^{\prime}\right) \eta\left(t, t^{\prime} ; t_{\times}\right)
\end{gathered}
$$

The relationship of these identities to the common equilibrium Ward identity is not apparent. In the time domain, the previously reported equation denoted as Ward identity was given in [30]. In [49], it was obtained from our Ward identity (46) as its integral over $t_{\times}$. The same integration can be performed in the present case and it results into the Strinati identity again:

$$
\int \mathrm{d} t_{\times} \boldsymbol{\Delta}\left(t, t^{\prime} ; t_{\times}\right)=-\mathrm{i}\left(t-t^{\prime}\right) \boldsymbol{\Sigma}\left(t, t^{\prime}\right)
$$

Only it has to be remembered that all times must exceed $t_{\mathrm{I}}$ now. The less-component is special. By Eq. (47),

$$
\begin{array}{r}
\int \mathrm{d} t_{\times} \Delta^{<}\left(t, t^{\prime} ; t_{\times}\right)=-\mathrm{i}\left\{\left(t-t^{\prime}\right) ._{\bullet}^{<}\left(t, t^{\prime}\right)\right. \\
\left.+\left(t-t_{\mathrm{I}}\right) . \Sigma_{\circ}^{<}\left(t, t^{\prime}\right)-\left(t^{\prime}-t_{\mathrm{I}}\right){ }_{\circ} \Sigma_{\bullet}^{<}\left(t, t^{\prime}\right)\right\}
\end{array}
$$

A more illuminating comparison of Eq. (46) would be with the Ward identities in equilibrium. The equilibrium state itself lasts for ever. The Green's function for our process depends on the initial time, however. To achieve the steady state conditions, we have two options. Either we send the initial time $t_{\mathrm{I}}$ to $-\infty$. The singular terms in $\Sigma^{<}$will vanish for all finite times and the system will become homogeneous in time. Or we still start at a finite $t_{\mathrm{I}}$, but all times, $t, t^{\prime}, t_{\times}$, will be very large, so that the initial transient will be vanishingly small already. In either case, the vertex $\boldsymbol{\Delta}$ will depend on two time differences, say $\tau=t-t_{\times}$and $\tau^{\prime}=t^{\prime}-t_{\times}$, and its Fourier transform will have the well known form $[29,7,33]$,

$$
\begin{aligned}
\tilde{\boldsymbol{\Delta}}\left(\omega, \omega^{\prime}\right) & =\iint \mathrm{d} \tau \mathrm{d} \tau^{\prime} \boldsymbol{\Delta}\left(\tau, \tau^{\prime} ; 0\right) \cdot \mathrm{e}^{\mathrm{i}\left(\omega \tau-\omega^{\prime} \tau^{\prime}\right)} \\
& =-\frac{\boldsymbol{\Sigma}(\omega)-\boldsymbol{\Sigma}\left(\omega^{\prime}\right)}{\omega-\omega^{\prime}}
\end{aligned}
$$

which completes the identification.

\subsection{Ward identity, reconstruction equations, Ansatzes}

The equation (42) is written for the linear variation of the Green's function under the effect of an external field. It may be rewritten in terms of functional derivatives, that is three-terminal quantities directly related to the response of the system and its transport properties:

$$
\begin{aligned}
\frac{\delta \mathbf{G}}{\delta \mathcal{U}} & =\mathbf{G}\left(\boldsymbol{\Gamma}_{0}+\frac{\delta \boldsymbol{\Sigma}}{\delta \mathcal{U}}\right) \mathbf{G} \\
\boldsymbol{\Gamma}_{0}\left(1,1^{\prime} ; 2\right) & =\frac{\delta \mathcal{U}(1)}{\delta \mathcal{U}(2)} \delta\left(1-1^{\prime}\right)
\end{aligned}
$$


The basic quantity is the vertex correction to the bare vertex $\boldsymbol{\Gamma}_{0}$ coupling the system to external fields [50].

The virtue of the Ward identity (46) valid for the special case of spatially homogeneous fields is to reduce the three-terminal vertex to the two-terminal self-energy; the three-point structure is imposed by the scalar function $\eta\left(t, t^{\prime} ; t_{\times}\right)$. The functional derivative of $\mathbf{G}$ has the same structure for homogeneous fields; the last equation thus reduces to

$$
\begin{aligned}
& \mathbf{G}\left(t, t^{\prime}\right) \eta\left(t, t^{\prime} ; t_{\times}\right) \\
& =\mathrm{i} \mathbf{G}\left(t, t_{\times}\right) \mathbf{G}\left(t_{\times}, t^{\prime}\right) \eta\left(t, t^{\prime} ; t_{\times}\right) \\
& +\iint \mathrm{d} \bar{t} \mathrm{~d} \overline{\bar{t}} \mathbf{G}(t, \bar{t}) \mathbf{\Sigma}(\bar{t}, \overline{\bar{t}}) \eta\left(\bar{t}, \overline{\bar{t}} ; t_{\times}\right) \mathbf{G}\left(\overline{\bar{t}}, t^{\prime}\right)
\end{aligned}
$$

This equation, in contrast to Eq. (51), involves oneparticle (two-point) quantities only. It is a condition governing the matrix Green's function; from the derivation, it is clear that it serves as a necessary condition for the Ward identity, and, hence, for the particle number conservation. It is valid for the exact Green's function as a tautology, but it also holds for any self-consistent approximation of the self-energy.

There are two different uses of Eq. (52). The first and obvious way of using it is to check any particular physical approximation for its internal consistency, for example, to verify that all components of the self-energy are treated in a uniform manner.

Next, we come to the second, not so widely known, use of Eq. (52). It should be recalled that in our approach to quantum transport, we insisted on a separation of two "orthogonal" kinds of approximations, the underlying physical approximation for the treatment of interactions, and the approximations of the Ansatz type simplifying the dynamical aspects of the theory with the aim at a quantum transport equation. The identity (52) finds its second, complementary use in judging also this other class of approximations. To get a better insight, it is convenient to write it by components. Non-zero results are obtained only for two arrangements of times. First, for $t>t_{\times}>t^{\prime}$, two equations are obtained from Eq. (52), one for the retarded propagator, the other for the particle correlation function. The first of these equations is identical with the renormalized semi-group rule (33). The second one,

$$
\begin{aligned}
& G^{<}\left(t, t^{\prime}\right)=\mathrm{i} G^{R}\left(t, t_{\times}\right) \cdot G^{<}\left(t_{\times}, t^{\prime}\right) \\
& +\int_{t_{\times}}^{t} \mathrm{~d} \bar{t} \int_{t_{\mathrm{I}}}^{t_{\times}} \mathrm{d} \overline{\bar{t}} G^{R}(t, \bar{t}) \Sigma^{R}(\bar{t}, \overline{\bar{t}}) G^{<}\left(\overline{\bar{t}}, t^{\prime}\right) \\
& +\int_{t_{\times}}^{t} \mathrm{~d} \bar{t} \int_{t_{\mathrm{I}}}^{t_{\times}} \mathrm{d} \overline{\bar{t}} G^{R}(t, \bar{t}) \Sigma^{<}(\bar{t}, \overline{\bar{t}}) G^{A}\left(\overline{\bar{t}}, t^{\prime}\right)
\end{aligned}
$$

is a "mixed" composition rule for the product $G^{R} G^{<}$. This equation should be compared with the reconstruction equation (15). The latter is nothing but a limiting case obtained from (53) by merging the two earlier times, $t_{\times} \rightarrow t^{\prime}$.

For the reverse order of times, $t<t_{\times}<t^{\prime}$, another two relations result from (52). One is the analogue of (33) for the advanced propagator $G^{A}$. The other one, for $G^{<}$, is symmetric to (53); we will refer to them as to $(33 ')$ and (53') without writing them down explicitly.

The outcome of this analysis is that the Green's function has to satisfy the original reconstruction equations exactly in order to obey the Ward identity. Any Ansatz is conceived as an approximation neglecting some of the vertex corrections in the reconstruction equations. Our objective should be to minimize the inaccuracy associated with the application of an Ansatz.

Similar considerations should be paid to any violation of the renormalized semi-group rule (33).

This provides a more rigorous framework for the task of weighing the reliability of various Ansatzes opened in Sec. 6.5. There, we suggested that the standard GKBA literally taken will be suffering from taking the vertex corrections insufficiently into account. This was the reason for attempting the alternative quasi-particle scheme leading to QKBA and in the future to RQKBA. The results of this section gave these alternatives full justification in the case that decay of correlations is much faster than any other physical process in the system. Using the quasi-particle picture, we were then able to satisfy the renormalized QCR almost exactly. In the QKBA, the polaron term was suppressed, while the irreducible vertex still remained untreated. Incorporation of this term by a proper renormalization of quasi-particle propagators accounting for particle interactions will be left to the proposed RQKBA. Meanwhile, there is always the option of treating these corrections in a phenomenological way. This has met with practical success already $[51-53,8,9]$; there remains the problem of adjustable parameters selected ad hoc. 


\section{Summary}

This review deals with two distinct but closely interwoven themes, both concerning the use of Nonequilibrium Green's functions as a formalism for description of quantum electronic transport processes starting from complex correlated initial conditions and driven by rapidly changing external disturbances arbitrarily far into non-equilibrium. The first theme is an actual technical possibility of taking the correlated initial conditions into account. On the general NGF level, there are several possibilities, to which we add an alternative of generating the correlated initial state in the course of a preparatory real time process of the Keldysh switch-on type. The advantage of this approach is that all such initial states just exhaust the states natural in physical sense. We further derive the transport Ward identity incorporating the correlated initial conditions. It bridges both main themes, as it leads on the reconstruction equations for the Keldysh initial states. The second theme addresses a general scheme of developing quantum transport equations for the single-particle distribution function. This scheme has an exact general part, in which the Dyson equation for the particle correlation function is transformed into a coupled pair of equations, the precursor transport equation and the reconstruction equations. The correlated initial conditions enter both. An approximate transition to the quantum transport equation level depends on the hierarchy of characteristic times. It appears as possible, if the decay of correlations is faster than both the relaxation of the electron distribution and the variation of the external disturbances in time - in justification of the Bogolyubov conjecture. The same conditions guarantee formation of the quasi-particle mode of dynamics of the system; the two results are, in fact, intimately connected. In the regime thus defined, we may say that a universal theoretical scheme for studying quantum transport has been built up. This scheme permits to study the earliest stages of a transient process using the full NGF description as well as the later stages of development using the quantum transport equations having the long time asymptotic character.

The scheme is now ready for use; one attempt is presented in the parallel paper [34]. There are interesting physical situations outside the above conditions; they would require an extension of the present approach. One would be an anomalously long decay of correlations caused by threshold or bottleneck phenomena in the decay channels. Another case would be a rapid variation of the external fields. For example, the field amplitude may vary moderately in time with intermittent jumps. If the jumps were faster than the decay of correlations, we might consider each of them as a renewed process with another initial state treated in the same manner as the original initial state.

\section{Acknowledgments}

This research was partially supported by the Grant Agency of the Czech Republic within the grant project $202 / 08 / 0361$. The research was carried out by V.S. within the Institutional Research Plan AV0Z10100521, by A.K. within the Institutional Research Plan AV0Z10100520, both financed by the Academy of Sciences of the Czech Republic, and by B.V. within the Institutional Research Plan MSM0021620834 financed by the Ministry of Education, Youth and Sports of the Czech Republic.

\section{References}

[1] L. P. Kadanoff and G. Baym, Quantum Statistical Mechanics, Benjamin, New York, 1962.

[2] L.V. Keldysh, Zh. Exp. Teor. Fiz. 47 (1964) 1515. [Sov. Phys. JETP 20 (1965) 1018 ]

[3] D. C. Langreth and G. Wilkins, Phys. Rev. B 6 (1972) 3189 .

[4] D. C. Langreth, in Linear and Nonlinear Electron Transport in Solids (J. T. Devreese and E. van Boren, eds.), Plenum, New York, 1976.

[5] E.M. Lifshitz, L.P. Pitaevskii, Physical Kinetics, L.D.Landau and E.M. Lifshitz Course of Theoretical Physics, Vol.10, Pergamon Press 1981.

[6] J. Rammer, H. Smith, Rev. Mod. Phys. 58 (1986) 323.

[7] G.D. Mahan, Many Particle Physics, (Plenum Press, New York, 1990).

[8] H. Haug, A.P. Jauho, Quantum Kinetics in Transport and Optics of Semiconductors, Springer, Berlin, 1997.

[9] M. Bonitz, Quantum Kinetic Theory, Teubner, Stuttgart, 1998. 
[10] V. Špička, B. Velický, A. Kalvová, Physica E 29 (2005) 154, ibid 175, ibid 196.

[11] B. Velický, A. Kalvová, V. Špička, J.Phys: Conference Series 35 (2006) 1.

[12] M. Bonitz, A. Filinov (eds.), Progress in Nonequilibrium Green's Functions III, J.Phys: Conference Series 35, 2006.

[13] M. Bonitz, D. Semkat (eds.), Introduction to Computational Methods in Many Body Physics, Rinton Press, Inc., New Jersey 2006

[14] E. Runge and E.K.U. Gross, Phys. Rev. Lett. 52 (1984) 997

[15] K. Burke, E.K.U. Gross, A Guided Tour of TimeDependent Density Functional Theory in D. Joubert(ed), Density Functionals: Theory and Applications, Lecture Notes in Phys. 500, Springer, 1998, pp. 116

[16] R. Van Leeuwen, Int. J. Mod. Phys. B 15 (2001) 1969.

[17] G. Onida, L. Reining, and A. Rubio, Rev. Mod. Phys. 74 (2002) 601.

[18] R. van Leeuwen, N.E. Dahlen, G. Stefanucci, C.O. Almbladh, and U. von Barth, cond-mat/0506130.

[19] A. R. Rocha, V. M. Garca-Surez, S. Bailey, C. Lambert, J. Ferrer, and S. Sanvito, Phys. Rev. B 73 (2006) 085414.

[20] N.E. Dahlen, R. van Leeuwen, Phys. Rev. Lett. 98 (2007) 153004.

[21] K. S. Thygesen, A. Rubio, Phys. Rev. B 73 (2008) 115333.

[22] N.N. Bogolyubov, in Studies in Statistical Mechanics, in G. Uhlenbeck and J. DeBoer (eds), Studies in Statistical Mechanics, Vol.1, Amsterdam, 1962.

[23] G.V. Chester, Rep. Prog. Phys. 26 (1963) 411.

[24] A.I. Akhiezer and S.V. Peletminskij, Methods of Statistical Physics, Pergamon, N.Y. 1980.

[25] P. Lipavský, V. Špička, and B. Velický Phys. Rev. B 29 (1986) 6933 .

[26] A.V. Vinogradov, Trudy Fiz. Inst. Im. P.N. Lebedeva 187(1988) 117.

[27] B. Velický, A. Kalvová, Phys. Stat. Sol.(b) 188 (1995) 515.

[28] B. Velický, A. Kalvová and V. Špička, Phys. Rev. B 75 (2007) 195125 .

[29] B. Velický, Phys. Rev. 184, 614 (1969).

[30] G. Strinati, Riv. Il Nuovo Cim. 11, 1 (1988).

[31] M. Revzen, T. Toyoda, Y. Takahashi, F.C. Khanna, Phys. Rev. B, 769 (1989).

[32] A. Gonis, Theoretical Materials Science (Materials Research Society, Warrendale, 2000)
[33] R. Ramazashvili, Phys. Rev. B 66, 220503(R) (2002).

[34] B. Velický, A. Kalvová, V. Špička, these Proceedings

[35] S. Fujita, Phys. Rev. A 4 (1971) 1114.

[36] A.G. Hall, J. Phys. A 8 (1975) 214.

[37] Yu.A. Kukharenko, S.G. Tikhodeev, Soviet. Phys. JETP 56 (1982), 831.

[38] P. Danielewicz, Ann. Phys. (N.Y.) 152 (1984) 239.

[39] M. Wagner, Phys. Rev. B 44 (1991) 6104.

[40] D. Semkat, D. Kremp, and M. Bonitz, Phys. Rev. E 59 (1999) 1557.

[41] V.G. Morozov, G. Ropke, Ann. Phys. 278 (1999) 127.

[42] D. Semkat, D. Kremp, and M. Bonitz, J. Math. Phys. 41 (2000) 7458.

[43] K. Morawetz, M. Bonitz, V.G. Morozov, G. Ropke, and D. Kremp Phys. Rev. E bf 63 (2001) 020102-1.

[44] D. Semkat, D. Kremp, and M. Bonitz, Contrib. Plasma Phys. 43 (2003) 321.

[45] D. Kremp, D. Semkat and M. Bonitz, J. Phys.: Conference Series 11(2005) 1.

[46] A. P. Jauho, N.S. Wingreen and Y. Meir, Phys. Rev. B 50 (1994) 5528.

[47] A. Messiah, Quantum Mechanics, Dover, 1999.

[48] B. Velický, A. Kalvová and V. Špička, Phys. Rev. B (2009), to appear.

[49] B. Velický, A. Kalvová and V. Špička, Phys. Rev. B 77 (2008) 041201 (R).

[50] For any self-consistent approximation for self-energy, $\boldsymbol{\Sigma}=\boldsymbol{\Sigma}[\mathbf{G}]$, the vertex may be rearranged using the chain rule for derivatives and (51) yields the BetheSalpeter equation

$$
\frac{\delta \mathbf{G}}{\delta \mathcal{U}}=\mathbf{G}\left[\boldsymbol{\Gamma}_{0}+\frac{\delta \boldsymbol{\Sigma}}{\delta \mathbf{G}} \frac{\delta \mathbf{G}}{\delta \mathcal{U}}\right] \mathbf{G}
$$

[51] H. Haug, Phys. Stat. Sol. B 173 (1992) 139.

[52] L. Banyai, B.B. Tran Thoai, C. Remling, H. Haug, Phys. Stat. Sol. B 173 (1992) 149.

[53] H. Haug, L. Banyai, Solid State Comm. 100 (1996) 303. 